\title{
TRIDIAGONAL PAIRS OF TYPE III WITH HEIGHT ONE*
}

\author{
XUE $\mathrm{LI}^{\dagger}, \mathrm{BO} \mathrm{HOU}^{\ddagger}$, AND SUOGANG $\mathrm{GAO}^{\S}$
}

\begin{abstract}
Let $\mathbb{K}$ denote an algebraically closed field with characteristic 0 . Let $V$ denote a vector space over $\mathbb{K}$ with finite positive dimension, and let $A, A^{*}$ denote a tridiagonal pair on $V$ of diameter $d$. Let $V_{0}, \ldots, V_{d}$ denote a standard ordering of the eigenspaces of $A$ on $V$, and let $\theta_{0}, \ldots, \theta_{d}$ denote the corresponding eigenvalues of $A$. It is assumed that $d \geq 3$. Let $\rho_{i}$ denote the dimension of $V_{i}$. The sequence $\rho_{0}, \rho_{1}, \ldots, \rho_{d}$ is called the shape of the tridiagonal pair. It is known that $\rho_{0}=1$ and there exists a unique integer $h(0 \leq h \leq d / 2)$ such that $\rho_{i-1}<\rho_{i}$ for $1 \leq i \leq h, \rho_{i-1}=\rho_{i}$ for $h<i \leq d-h$, and $\rho_{i-1}>\rho_{i}$ for $d-h<i \leq d$. The integer $h$ is known as the height of the tridiagonal pair. In this paper, it is showed that the shape of a tridiagonal pair of type III with height one is either $1,2,2, \ldots, 2,1$ or $1,3,3,1$. In each case, an interesting basis is found for $V$ and the actions of $A, A^{*}$ on this basis are described.
\end{abstract}

Key words. Tridiagonal pair, Tridiagonal relation, Height, Shape.

AMS subject classifications. 05E30, 05E35, 33C45, 33D45.

1. Introduction. Throughout the paper, $\mathbb{K}$ denotes an algebraically closed field with characteristic 0 and $V$ denotes a vector space over $\mathbb{K}$ with finite positive dimension.

We begin by recalling the notion of a tridiagonal pair. We will use the following terms. Let $\operatorname{End}(V)$ denote the $\mathbb{K}$-algebra consisting of all $\mathbb{K}$-linear transformations from $V$ to $V$. For $A \in \operatorname{End}(V)$ and for a subspace $W \subseteq V$, we call $W$ an eigenspace of $A$ whenever $W \neq 0$ and there exists $\theta \in \mathbb{K}$ such that $W=\{v \in V \mid A v=\theta v\}$; in this case, $\theta$ is the eigenvalue of $A$ associated with $W$. We say $A$ is diagonalizable whenever $V$ is spanned by the eigenspaces of $A$.

Definition 1.1. ([1, Definition 1.1]) By a tridiagonal pair on $V$, we mean an ordered pair $A, A^{*} \in$ $\operatorname{End}(V)$ that satisfy (i)-(iv) below:

(i) Each of $A, A^{*}$ is diagonalizable on $V$.

(ii) There exists an ordering $\left\{V_{i}\right\}_{i=0}^{d}$ of the eigenspaces of $A$ such that

$$
A^{*} V_{i} \subseteq V_{i-1}+V_{i}+V_{i+1} \quad(0 \leq i \leq d),
$$

where $V_{-1}=0$ and $V_{d+1}=0$.

(iii) There exists an ordering $\left\{V_{i}^{*}\right\}_{i=0}^{\delta}$ of the eigenspaces of $A^{*}$ such that

$$
A V_{i}^{*} \subseteq V_{i-1}^{*}+V_{i}^{*}+V_{i+1}^{*} \quad(0 \leq i \leq \delta)
$$

where $V_{-1}^{*}=0$ and $V_{\delta+1}^{*}=0$.

\footnotetext{
*Received by the editors on August 9, 2017. Accepted for publication on August 5, 2019. Handling Editor: Bryan L. Shader. Corresponding Author: Suogang Gao.

${ }^{\dagger}$ College of Mathematics and Information Science, Hebei Normal University, Shijiazhuang 050024, P.R. China, and Center for Combinatorics, LPMC-TJKLC Nankai University, Tianjin 300071, P.R. China (550668549@qq.com).

${ }^{\ddagger}$ College of Mathematics and Information Science, Hebei Normal University, Shijiazhuang 050024, P.R. China (houbo1969@163.com).

$\S$ College of Mathematics and Information Science, Hebei Normal University, Shijiazhuang 050024, P.R. China (sggaomail@163.com,sggao@hebtu.edu.cn).
} 
(iv) There is no subspace $W$ of $V$ such that both $A W \subseteq W, A^{*} W \subseteq W$, other than $W=0$ and $W=V$.

We say the pair $A, A^{*}$ is over $\mathbb{K}$.

Let $A, A^{*}$ denote a tridiagonal pair on $V$. By [1, Lemma 4.5], the integers $d$ and $\delta$ from Definition 1.1 are equal; we call this common value the diameter of $A, A^{*}$. We assume that $d \geq 3$. An ordering of the eigenspaces of $A$ (resp., $A^{*}$ ) is said to be standard whenever it satisfies (1.1) (resp., (1.2)). By [1, Corollary 5.7], for $0 \leq i \leq d$, the spaces $V_{i}, V_{i}^{*}$ have the same dimension; we denote this common dimension by $\rho_{i}$. We call the sequence $\left\{\rho_{i}\right\}_{i=0}^{d}$ the shape of $A, A^{*}$. By a Leonard pair we mean a tridigonal pair with shape $(1,1, \ldots, 1)\left[8\right.$, Definition 1.1]. See [5, 8, 9] for more information about Leonard pairs. The pair $A, A^{*}$ is said to be sharp whenever $\rho_{0}=1$. By [7, Theorem 1.3], a tridiagonal pair over an algebraically closed field is sharp. See $[2,6]$ for more information about sharp tridiagonal pairs. By [1, Corollaries 5.7 and 6.6], $\left\{\rho_{i}\right\}_{i=0}^{d}$ is symmetric and unimodal; that is $\rho_{i}=\rho_{d-i}$ for $0 \leq i \leq d$ and $\rho_{i-1} \leq \rho_{i}$ for $1 \leq i \leq \frac{d}{2}$. By [3, Theorem 3.3], there exists a unique integer $h$ with $0 \leq h \leq \frac{d}{2}$ such that $\rho_{i-1}<\rho_{i}$ for $1 \leq i \leq h, \rho_{i-1}=\rho_{i}$ for $h<i \leq d-h$ and $\rho_{i-1}>\rho_{i}$ for $d-h<i \leq d$. We call $h$ the height of $A, A^{*}$.

It is known [1, Theorem 10.1] that there exists a sequence of scalars $\beta, \gamma, \gamma^{*}, \varrho, \varrho^{*}$ taken from $\mathbb{K}$ such that both

$$
\begin{aligned}
& {\left[A, A^{2} A^{*}-\beta A A^{*} A+A^{*} A^{2}-\gamma\left(A A^{*}+A^{*} A\right)-\varrho A^{*}\right]=0,} \\
& {\left[A^{*}, A^{*^{2}} A-\beta A^{*} A A^{*}+A A^{*^{2}}-\gamma^{*}\left(A^{*} A+A A^{*}\right)-\varrho^{*} A\right]=0,}
\end{aligned}
$$

where $[B, C]=B C-C B$. The sequence is unique if the diameter of the pair is at least 3 . The above relations are known as the tridiagonal relations. The scalar $\beta$ is called the fundamental parameter of $A, A^{*}$. A tridiagonal pair $A, A^{*}$ is called of type I, type II, type III according to $\beta \neq \pm 2, \beta=2, \beta=-2$ in the tridiagonal relations, respectively.

In [4], K. Nomura considered tridiagonal pairs $A, A^{*}$ of type I with height one. He showed that the shape of a tridiagonal pair of height one is either $1,2,2, \ldots, 2,1$ or $1,3,3,1$. And he gave a basis for $V$ and obtained the matrices representing $A, A^{*}$ with respect to this basis. Motivated by [4], in this paper, we show that the shape of a tridiagonal pair of type III with height one is the same as type I in [4], that is, the shape is either $1,2,2, \ldots, 2,1$ or $1,3,3,1$. Then, in each case, we display a basis for $V$ and give the actions of $A, A^{*}$ on this basis.

In Section 2, we first recall some basic results from [1] and [3] concerning the split decomposition and the refined split decomposition of the tridiagonal pair. In Section 3, we define some linear transformations $X_{i}, Y_{i}, Z_{i}(0 \leq i \leq d)$, and use them to show that $R L^{(+)}$(resp., $\left.R L^{(-)}\right)$is a scalar multiple of $L^{(+)} R$ (resp., $L^{(-)} R$ ) on each $U^{(r)} \cap U_{i}$. We also give a linear relation between $R L^{(0)}, L^{(0)} R$ and $I$ (the identity map) on each $U^{(r)} \cap U_{i}$. In Section 4, we find a basis for $V$. In Section 5 , we obtain the action of $L$. In Section 6 , we determine the shape of the tridiagonal pair of type III with height 1, which is Theorem 6.4. In Section 7, for all cases listed in Theorem 6.4, we display a basis for $V$ and give the actions of $A, A^{*}$ on this basis.

2. Refined split decomposition of a tridiagonal pair. In this section, we first recall some known facts about the split decomposition and the refined split decomposition of the tridaigonal pair.

With reference to Definition 1.1, for $0 \leq i \leq d$, we set

$$
U_{i}=\left(V_{0}^{*}+V_{1}^{*}+\cdots+V_{i}^{*}\right) \cap\left(V_{i}+V_{i+1}+\cdots+V_{d}\right) .
$$


By [1, Corollary 5.7], the dimension of $U_{i}$ is equal to $\rho_{i}$ for $0 \leq i \leq d$. And by [1, Theorem 4.6], the space $V$ is decomposed as

$$
V=U_{0}+U_{1}+\cdots+U_{d} \quad(\text { direct sum })
$$

The decomposition given in (2.3) is called the split decomposition of the tridiagonal pair.

Let $F_{i}: V \rightarrow U_{i}$ denote the projection with respect to the direct sum (2.3). Then, for $0 \leq i, j \leq d$,

$$
F_{0}+F_{1}+\cdots+F_{d}=I \text { and } F_{i} F_{j}=\delta_{i j} F_{i}
$$

The raising map $R$ and the lowering map $L$ are defined as follows:

$$
R=A-\sum_{i=0}^{d} \theta_{i} F_{i} \quad \text { and } \quad L=A^{*}-\sum_{i=0}^{d} \theta_{i}^{*} F_{i}
$$

Lemma 2.1. ([1, Corollary 6.3])

(i) $R U_{i} \subseteq U_{i+1}(0 \leq i<d), R U_{d}=0$.

(ii) $L U_{i} \subseteq U_{i-1}(0<i \leq d), L U_{0}=0$.

Lemma 2.2. ([3, Lemma 2.7]) Let $W$ denote a subspace of $V$. Suppose that $R W \subseteq W, L W \subseteq W$ and $F_{i} W \subseteq W$ for $0 \leq i \leq d$. Then $W=0$ or $W=V$.

Next, we display some results concerning the refined split decomposition of the tridiagonal pair. For the rest of this paper, let $h$ denote the height of the tridiagonal pair.

For $0 \leq r \leq h$ and $r \leq i \leq d-r$, we set

$$
U_{i}^{(r)}=R^{i-r}\left(U_{r} \cap \operatorname{Ker} R^{d-2 r+1}\right) .
$$

Lemma 2.3. ([3, Lemma 4.1]) For $0 \leq r \leq h$, the following hold.

(i) $U_{0}^{(0)}=U_{0}$ and $U_{d}^{(0)}=U_{d}$,

(ii) $U_{i}^{(r)} \subseteq U_{i}(r \leq i \leq d-r)$,

(iii) $U_{r}^{(r)}=U_{r} \cap \operatorname{Ker} R^{d-2 r+1}$,

(iv) $U_{i}^{(r)}=R^{i-r} U_{r}^{(r)}(r \leq i \leq d-r)$,

(v) $R U_{i}^{(r)}=U_{i+1}^{(r)}(r \leq i \leq d-r-1), R U_{d-r}^{(r)}=0$,

(vi) The restriction $\left.R\right|_{U_{i}^{(r)}}$ : $U_{i}^{(r)} \rightarrow U_{i+1}^{(r)}$ is a bijection $(r \leq i \leq d-r-1)$.

Lemma 2.4. ([3, Lemma 4.3]) For $0 \leq r \leq h$,

$$
\operatorname{dim} U_{i}^{(r)}=\rho_{r}-\rho_{r-1} \quad(r \leq i \leq d-r),
$$

where we set $\rho_{-1}=0$.

Lemma 2.5. ([3, Lemmas 4.7 and 4.8]) For $0 \leq i \leq d$,

$$
\left.U_{i}=\sum_{r=0}^{m} U_{i}^{(r)} \quad \text { (direct sum }\right),
$$


where $m=\min \{i, h, d-i\}$. And $V$ is decomposed as

$$
\left.V=\sum_{r=0}^{h} \sum_{i=r}^{d-r} U_{i}^{(r)} \quad \text { (direct sum }\right)
$$

The decomposition given in (2.4) is called the refinement split decomposition of the tridiagonal pair.

For $0 \leq r \leq h$, we set

$$
U^{(r)}=\sum_{i=r}^{d-r} U_{i}^{(r)}
$$

Lemma 2.6. ([3, Lemma 5.1]) $V$ is decomposed as

$$
V=\sum_{r=0}^{h} U^{(r)} \quad \text { (direct sum). }
$$

Lemma 2.7. ([3, Lemma 5.3]) For $0 \leq r \leq h$,

$$
R U^{(r)} \subseteq U^{(r)} .
$$

Let

$$
F^{(r)}: V \rightarrow U^{(r)} \quad(0 \leq r \leq h)
$$

denote the projection with respect to the direct sum $V=\sum_{r=0}^{h} U^{(r)}$. Then, for $0 \leq r \leq h$ and $0 \leq s \leq h$,

$$
F^{(0)}+F^{(1)}+\cdots+F^{(h)}=I \quad \text { and } \quad F^{(r)} F^{(s)}=\delta_{r s} F^{(r)} .
$$

We set

$$
F_{i}^{(r)}=F_{i} F^{(r)} \quad(0 \leq r \leq h, 0 \leq i \leq d) .
$$

Lemma 2.8. ([3, Lemma 6.1]) For $0 \leq r \leq h$ and $0 \leq i \leq d$,

(i) $F_{i}^{(r)}=F^{(r)} F_{i}=F_{i} F^{(r)}$,

(ii) $F_{0}^{(0)}=F_{0}$ and $F_{d}^{(0)}=F_{d}$,

(iii) $F_{i}^{(r)} \neq 0$ if and only if $r \leq i \leq d-r$.

Lemma 2.9. ([3, Lemma 6.2]) For $0 \leq r \leq h$ and $r \leq i \leq d-r, F_{i}^{(r)} V=U_{i}^{(r)}$, and

$$
F_{i}^{(r)}: V \rightarrow U_{i}^{(r)}
$$

is the projection with respect to the direct sum $V=\sum_{r=0}^{h} \sum_{i=r}^{d-r} U_{i}^{(r)}$.

Lemma 2.10. ([3, Lemma 6.3]) For $0 \leq r \leq h$,

(i) $F^{(r)} R=R F^{(r)}$,

(ii) $F_{i}^{(r)} R=R F_{i-1}^{(r)}(1 \leq i \leq d)$,

(iii) $F_{r}^{(r)} R=0$.

We set

$$
L^{(-)}=\sum_{r=1}^{h} F^{(r-1)} L F^{(r)}, \quad L^{(0)}=\sum_{r=0}^{h} F^{(r)} L F^{(r)}, \quad L^{(+)}=\sum_{r=0}^{h-1} F^{(r+1)} L F^{(r)} .
$$


Lemma 2.11. ([3, Lemma 6.5])

$$
L=L^{(-)}+L^{(0)}+L^{(+)} .
$$

Lemma 2.12. ([3, Lemma 6.7]) For $0 \leq r \leq h$, the following hold.

(i) $L^{(0)} F_{r}^{(r)}=0$

(ii) $L^{(+)} F_{r}^{(r)}=L^{(+)} F_{r+1}^{(r)}=0$.

Let $A, A^{*}$ be a tridiagonal pair with the fundamental parameter $\beta$. For the rest of this paper, we always assume that $\beta=-2$.

Lemma 2.13. For $0 \leq i \leq d-2$,

$$
\begin{aligned}
\left(R^{3} L+R^{2} L R-R L R^{2}-L R^{3}-\varepsilon_{i} R^{2}\right) F_{i} & =0 \\
\left(L^{3} R+L^{2} R L-L R L^{2}-R L^{3}+\varepsilon_{i} L^{2}\right) F_{i+2} & =0
\end{aligned}
$$

where

$$
\varepsilon_{i}=\left(\theta_{i}-\theta_{i+2}\right)\left(\theta_{i+1}^{*}-\theta_{i+2}^{*}\right)-\left(\theta_{i+2}^{*}-\theta_{i}^{*}\right)\left(\theta_{i+1}-\theta_{i}\right) .
$$

Proof. Immediate from [1, Theorem 12.1] and $\beta=-2$.

LEMMA 2.14. The following relations hold.

$$
\begin{aligned}
& R^{3} L^{(+)}+R^{2} L^{(+)} R-R L^{(+)} R^{2}-L^{(+)} R^{3}=0, \\
& R^{3} L^{(-)}+R^{2} L^{(-)} R-R L^{(-)} R^{2}-L^{(-)} R^{3}=0 .
\end{aligned}
$$

Proof. Immediate from [3, Theorem 6.8] and $\beta=-2$.

Lemma 2.15. For $0 \leq r \leq h$ and $0 \leq i \leq d-2$,

$$
\left(R^{3} L^{(0)}+R^{2} L^{(0)} R-R L^{(0)} R^{2}-L^{(0)} R^{3}-\varepsilon_{i} R^{2}\right) F_{i}^{(r)}=0 .
$$

Proof. Immediate from [3, Lemma 6.9] and $\beta=-2$.

Lemma 2.16. ([1, Theorem 11.2]) Let $\left\{\theta_{i}\right\}_{i=0}^{d}$ (resp., $\left.\left\{\theta_{i}^{*}\right\}_{i=0}^{d}\right)$ denote an ordering of the eigenvalues of $A$ (resp., $\left.A^{*}\right)$. Then there exist scalars $a, a^{*}, b, b^{*}, c, c^{*}$ in $\mathbb{K}$ such that

$$
\begin{gathered}
\theta_{i}=a+b(-1)^{i}+c i(-1)^{i} \quad(0 \leq i \leq d), \\
\theta_{i}^{*}=a^{*}+b^{*}(-1)^{i}+c^{*} i(-1)^{i} \quad(0 \leq i \leq d) .
\end{gathered}
$$

3. Relations between $R$ and $L^{(+)}$(resp., $L^{(-)}, L^{(0)}$ ). In this section, we show that $R L^{(+)}$(resp., $R L^{(-)}$) is a scalar multiple of $L^{(+)} R$ (resp., $L^{(-)} R$ ) on each $U^{(r)} \cap U_{i}$. We also give a linear relation between $R L^{(0)}, L^{(0)} R$ and $I$ (the identity map) on each $U^{(r)} \cap U_{i}$.

We first fix an integer $r$ such that $0 \leq r \leq h-1$, and set

$$
X_{i}:=L^{(+)} F_{i}^{(r)} \quad(0 \leq i \leq d) .
$$

Lemma 3.1. $X_{r}=X_{r+1}=0$.

Proof. Immediate from Lemma 2.12 (ii). 
Lemma 3.2. For $r \leq i \leq d-r-3$,

$$
R^{3} X_{i}+R^{2} X_{i+1} R-R X_{i+2} R^{2}-X_{i+3} R^{3}=0 .
$$

Proof. From (2.8), we have

$$
\left(R^{3} L^{(+)}+R^{2} L^{(+)} R-R L^{(+)} R^{2}-L^{(+)} R^{3}\right) F_{i}^{(r)}=0 .
$$

Apply Lemma 2.10 to the above relation to obtain

$$
R^{3} L^{(+)} F_{i}^{(r)}+R^{2} L^{(+)} F_{i+1}^{(r)} R-R L^{(+)} F_{i+2}^{(r)} R^{2}-L^{(+)} F_{i+3}^{(r)} R^{3}=0 .
$$

Lemma 3.3. For $r+2 \leq i \leq d-r-1$,

$$
\begin{aligned}
& R X_{i}+X_{i+1} R=0, \quad \text { if } i-r \text { is even } \\
& \left(\begin{array}{c}
i-r+1 \\
2
\end{array}\right) R X_{i}+\left(\begin{array}{c}
i-r \\
2
\end{array}\right) X_{i+1} R=0, \quad \text { if } i-r \text { is odd. }
\end{aligned}
$$

Proof. Fix an integer $i(r+2 \leq i \leq d-r-1)$. By the definition of $X_{i}$ and Lemma 2.9, we have

$$
\left.R X_{i}\right|_{U_{i}^{(r)}} \neq 0,\left.\quad R X_{i}\right|_{U_{j}^{(r)}}=0(i \neq j),\left.\quad X_{i+1} R\right|_{U_{i}^{(r)}} \neq 0,\left.\quad X_{i+1} R\right|_{U_{j}^{(r)}}=0(i \neq j) .
$$

These imply that (3.14) and (3.15) hold on $V$ if and only if (3.14) and (3.15) hold on $U_{i}^{(r)}$.

We first show that (3.14) holds using induction on $i$. Setting $i=r$ in (3.13), and applying Lemma 3.1, we find

$$
-R X_{r+2} R^{2}-X_{r+3} R^{3}=-\left(R X_{r+2}+X_{r+3} R\right) R^{2}=0 .
$$

Note that

$$
\left.R^{2}\right|_{U_{r}^{(r)}}: U_{r}^{(r)} \rightarrow U_{r+2}^{(r)}
$$

is a bijection by Lemma 2.3. Remove the factor $(-1) R^{2}$ in (3.16) to obtain

$$
R X_{r+2}+X_{r+3} R=0 .
$$

Hence, (3.14) holds at $i=r+2$.

Now suppose (3.14) holds at $i-2$, where $i-2-r$ is a non-negative even integer. We will show that (3.14) holds at $i$. By the induction hypothesis,

$$
R X_{i-2}+X_{i-1} R=0 .
$$

Replacing $i$ by $i-2$ in (3.13),

$$
R^{3} X_{i-2}+R^{2} X_{i-1} R-R X_{i} R^{2}-X_{i+1} R^{3}=0 .
$$

Multiplying (3.18) by $(-1) R^{2}$ from the left,

$$
-R^{3} X_{i-2}-R^{2} X_{i-1} R=0 .
$$


Adding (3.19) and (3.20),

$$
-R X_{i} R^{2}-X_{i+1} R^{3}=0 .
$$

Since $\left.R^{2}\right|_{U_{i-2}^{(r)}}: U_{i-2}^{(r)} \rightarrow U_{i}^{(r)}$ is a bijection by Lemma 2.3, we can remove the factor $(-1) R^{2}$ to get

$$
R X_{i}+X_{i+1} R=0 .
$$

Hence, (3.14) holds.

Next, we show that (3.15) holds using induction on $i$. Setting $i=r+1$ in (3.13), and applying Lemma 3.1 , we obtain

$$
R^{2} X_{r+2} R-R X_{r+3} R^{2}-X_{r+4} R^{3}=0 .
$$

Since $\left.R\right|_{U_{r+1}^{(r)}}: U_{r+1}^{(r)} \rightarrow U_{r+2}^{(r)}$ is a bijection by Lemma 2.3, we can remove the factor $R$ to get

$$
R^{2} X_{r+2}-R X_{r+3} R-X_{r+4} R^{2}=0 .
$$

Multiplying (3.17) by $(-1) R$ from the left,

$$
-R^{2} X_{r+2}-R X_{r+3} R=0 .
$$

Adding (3.21) and (3.22),

$$
-2 R X_{r+3} R-X_{r+4} R^{2}=0 .
$$

Since $\left.R\right|_{U_{r+2}^{(r)}}: U_{r+2}^{(r)} \rightarrow U_{r+3}^{(r)}$ is a bijection by Lemma 2.3 , we may remove the factor $R$ to get

$$
\left(\begin{array}{l}
4 \\
2
\end{array}\right) R X_{r+3}+\left(\begin{array}{l}
3 \\
2
\end{array}\right) X_{r+4} R=0
$$

Hence, (3.15) holds at $i=r+3$.

Now suppose (3.15) holds at $i-2$, where $i-2-r$ is a non-negative odd integer. We will show that (3.15) holds at $i$. By the induction hypothesis,

$$
\left(\begin{array}{c}
i-r-1 \\
2
\end{array}\right) R X_{i-2}+\left(\begin{array}{c}
i-r-2 \\
2
\end{array}\right) X_{i-1} R=0
$$

Replacing $i$ by $i-2$ in (3.13),

$$
R^{3} X_{i-2}+R^{2} X_{i-1} R-R X_{i} R^{2}-X_{i+1} R^{3}=0 .
$$

Multiplying (3.24) by $\left(\begin{array}{c}i-r-1 \\ 2\end{array}\right)$,

$$
\left(\begin{array}{c}
i-r-1 \\
2
\end{array}\right) R^{3} X_{i-2}+\left(\begin{array}{c}
i-r-1 \\
2
\end{array}\right) R^{2} X_{i-1} R-\left(\begin{array}{c}
i-r-1 \\
2
\end{array}\right) R X_{i} R^{2}-\left(\begin{array}{c}
i-r-1 \\
2
\end{array}\right) X_{i+1} R^{3}=0 .
$$

Multiplying (3.23) by $(-1) R^{2}$ from the left,

$$
-\left(\begin{array}{c}
i-r-1 \\
2
\end{array}\right) R^{3} X_{i-2}-\left(\begin{array}{c}
i-r-2 \\
2
\end{array}\right) R^{2} X_{i-1} R=0 .
$$

Adding (3.25) and (3.26), since the restriction $\left.R\right|_{U_{i-2}^{(r)}}: U_{i-2}^{(r)} \rightarrow U_{i-1}^{(r)}$ is a bijection by Lemma 2.3, we can remove the factor $R$ to get

$$
(i-r-2) R^{2} X_{i-1}-\left(\begin{array}{c}
i-r-1 \\
2
\end{array}\right) R X_{i} R-\left(\begin{array}{c}
i-r-1 \\
2
\end{array}\right) X_{i+1} R^{2}=0 .
$$


Since $i-1-r$ is even, replacing $i$ by $i-1$ in (3.14), and then multiplying $-(i-r-2) R$ from the left, we obtain

$$
-(i-r-2) R^{2} X_{i-1}-(i-r-2) R X_{i} R=0 .
$$

Adding (3.27) and (3.28), since $\left.R\right|_{U_{i-1}^{(r)}}: U_{i-1}^{(r)} \rightarrow U_{i}^{(r)}$ is a bijection by Lemma 2.3, we can remove the factor $(-1) R$ to get

$$
\left(\begin{array}{c}
i-r+1 \\
2
\end{array}\right) R X_{i}+\left(\begin{array}{c}
i-r \\
2
\end{array}\right) X_{i+1} R=0
$$

Hence, (3.15) holds.

Theorem 3.4. For $0 \leq r \leq h-1$ and $r+2 \leq i \leq d-r-1$,

$$
\begin{aligned}
& \left(R L^{(+)}+L^{(+)} R\right) U_{i}^{(r)}=0, \quad \text { if } i-r \text { is even; } \\
& \left(R L^{(+)}+\frac{i-r-1}{i-r+1} L^{(+)} R\right) U_{i}^{(r)}=0, \quad \text { if } i-r \text { is odd } .
\end{aligned}
$$

Proof. Immediate from (3.14), (3.15) and the definition of $X_{i}$.

Next we fix an integer $r$ such that $1 \leq r \leq h$, and set

$$
Y_{i}:=L^{(-)} F_{i}^{(r)}(0 \leq i \leq d) \quad \text { and } \quad Y_{d+1}:=0 .
$$

LeMma 3.5. $Y_{d-r+1}=Y_{d-r+2}=0$.

Proof. Immediate from Lemma 2.8 (iii).

Lemma 3.6. For $r \leq i \leq d-r-1$,

$$
R^{3} Y_{i}+R^{2} Y_{i+1} R-R Y_{i+2} R^{2}-Y_{i+3} R^{3}=0 .
$$

Proof. Similar to the proof of (3.13).

LEMMA 3.7. For $r \leq i \leq d-r-1$,

$$
\begin{aligned}
& R Y_{i}+Y_{i+1} R=0, \quad \text { if } d-r-i \text { is odd; } \\
& \left(\begin{array}{c}
d-r-i+1 \\
2
\end{array}\right) R Y_{i}+\left(\begin{array}{c}
d-r-i+2 \\
2
\end{array}\right) Y_{i+1} R=0, \quad \text { if } d-r-i \text { is even. }
\end{aligned}
$$

Proof. We first show that (3.32) holds using induction on $i$. Applying Lemma 3.5, setting $i=d-r-1$ in (3.31) and then removing $R^{2}$ from the left, we obtain

$$
R Y_{d-r-1}+Y_{d-r} R=0 .
$$

Hence, (3.32) holds at $i=d-r-1$.

Now suppose (3.32) holds at $i+2$, where $d-r-i-2$ is a non-negative odd integer. We will show that (3.32) holds at $i$. By the induction hypothesis,

$$
R Y_{i+2}+Y_{i+3} R=0 .
$$

Multiplying (3.35) by $R^{2}$ from the right, adding (3.31) and then removing $R^{2}$ from the left, we can get (3.32). 
Next, we show that (3.33) holds using induction on $i$. Setting $i=d-r-2$ in (3.31) and applying Lemma 3.5 , we obtain

$$
R^{3} Y_{d-r-2}+R^{2} Y_{d-r-1} R-R Y_{d-r} R^{2}=0
$$

Multiplying (3.34) by $R$ on the two sides of the equation,

$$
R^{2} Y_{d-r-1} R+R Y_{d-r} R^{2}=0 .
$$

Adding (3.36) and (3.37), and then removing $R^{2}$, we have

$$
R Y_{d-r-2}+2 Y_{d-r-1} R=0 .
$$

Hence, (3.33) holds at $i=d-r-2$.

Now suppose (3.33) holds at $i+2$, where $d-r-i-2$ is a non-negative even integer. We will show that (3.33) holds at $i$. By the induction hypothesis,

$$
\left(\begin{array}{c}
d-r-i-1 \\
2
\end{array}\right) R Y_{i+2}+\left(\begin{array}{c}
d-r-i \\
2
\end{array}\right) Y_{i+3} R=0
$$

Multiplying (3.31) by $\left(\begin{array}{c}d-r-i \\ 2\end{array}\right)$, we have

$$
\left(\begin{array}{c}
d-r-i \\
2
\end{array}\right) R^{3} Y_{i}+\left(\begin{array}{c}
d-r-i \\
2
\end{array}\right) R^{2} Y_{i+1} R-\left(\begin{array}{c}
d-r-i \\
2
\end{array}\right) R Y_{i+2} R^{2}-\left(\begin{array}{c}
d-r-i \\
2
\end{array}\right) Y_{i+3} R^{3}=0
$$

Multiplying (3.38) by $R^{2}$ from the right, adding (3.39), and then removing $R$, we obtain

$$
\left(\begin{array}{c}
d-r-i \\
2
\end{array}\right) R^{2} Y_{i}+\left(\begin{array}{c}
d-r-i \\
2
\end{array}\right) R Y_{i+1} R-(d-r-i-1) Y_{i+2} R^{2}=0 .
$$

Since $d-r-i-1$ is odd, replacing $i$ by $i+1$ in (3.32), and then multiplying $(d-r-i-1) R$ from the right, we have

$$
(d-r-i-1) R Y_{i+1} R+(d-r-i-1) Y_{i+2} R^{2}=0 .
$$

Adding (3.40) and (3.41), and then removing $R$, we can get (3.33).

THEOREM 3.8. For $1 \leq r \leq h$ and $r \leq i \leq d-r-1$,

$$
\begin{aligned}
& \left(R L^{(-)}+L^{(-)} R\right) U_{i}^{(r)}=0, \quad \text { if } d-r-i \text { is odd } \\
& \left(R L^{(-)}+\frac{d-r-i+2}{d-r-i} L^{(-)} R\right) U_{i}^{(r)}=0, \quad \text { if } d-r-i \text { is even. }
\end{aligned}
$$

Proof. Immediate from (3.32), (3.33) and the definition of $Y_{i}$.

Finally we fix an integer $r$ such that $0 \leq r \leq h$, and set

$$
Z_{i}:=L^{(0)} F_{i}^{(r)} \quad(0 \leq i \leq d)
$$

LEMMA 3.9. $Z_{r}=Z_{d-r+1}=0$.

Proof. Immediate from Lemmas 2.8 (iii) and 2.12 (i). 
LEMMA 3.10. For $r \leq i \leq d-r-2$,

$$
R^{3} Z_{i}+R^{2} Z_{i+1} R-R Z_{i+2} R^{2}-Z_{i+3} R^{3}-\varepsilon_{i} R^{2} F_{i}^{(r)}=0 .
$$

Proof. Immediate from (2.10) and Lemma 2.10.

Lemma 3.11. For $r+1 \leq i \leq d-r-1$,

$$
\begin{gathered}
\frac{i-r+1}{2} R^{2} Z_{i}-R Z_{i+1} R-\frac{i-r+1}{2} Z_{i+2} R^{2} \\
-\sum_{j=r}^{i-1}\left[\frac{1}{4}-\frac{1}{4}(-1)^{j-r+1}+\frac{j-r+1}{2}(-1)^{j-r}\right] \varepsilon_{j} R F_{i}^{(r)}=0, \quad \text { if } i-r \text { is odd } ; \\
\frac{i-r+2}{2} R^{2} Z_{i}-\frac{i-r}{2} Z_{i+2} R^{2}+\sum_{j=r}^{i-1}\left[\frac{1}{4}-\frac{1}{4}(-1)^{j-r+1}+\frac{j-r+1}{2}(-1)^{j-r}\right] \varepsilon_{j} R F_{i}^{(r)}=0, \\
\text { if } i-r \text { is even. }
\end{gathered}
$$

Proof. We show that the results hold using induction on $i$. Setting $i=r$ in (3.44), and applying Lemma 3.9 , we find

$$
R^{2} Z_{r+1} R-R Z_{r+2} R^{2}-Z_{r+3} R^{3}-\varepsilon_{r} R^{2} F_{r}^{(r)}=0 .
$$

Using Lemma 2.10 and removing $R$, we obtain

$$
R^{2} Z_{r+1}-R Z_{r+2} R-Z_{r+3} R^{2}-\varepsilon_{r} R F_{r+1}^{(r)}=0 .
$$

Hence, (3.45) holds at $i=r+1$.

Now suppose the results hold at $i-1$, where $i-1-r$ is a non-negative odd integer. We will show that the results hold at $i$. By the induction hypothesis,

$$
\frac{i-r}{2} R^{2} Z_{i-1}-R Z_{i} R-\frac{i-r}{2} Z_{i+1} R^{2}-\sum_{j=r}^{i-2}\left[\frac{1}{4}-\frac{1}{4}(-1)^{j-r+1}+\frac{j-r+1}{2}(-1)^{j-r}\right] \varepsilon_{j} R F_{i-1}^{(r)}=0 .
$$

Replacing $i$ by $i-1$ in (3.44), and multiplying by $\frac{i-r}{2}$,

$$
\frac{i-r}{2} R^{3} Z_{i-1}+\frac{i-r}{2} R^{2} Z_{i} R-\frac{i-r}{2} R Z_{i+1} R^{2}-\frac{i-r}{2} Z_{i+2} R^{3}-\frac{i-r}{2} \varepsilon_{i-1} R^{2} F_{i-1}^{(r)}=0 .
$$

Multiplying (3.48) by (-1) $R$ from the left, adding (3.49), and then removing $R$, we can get (3.46).

Next, we suppose the results hold at $i-1$, where $i-1-r$ is a non-negative even integer. We will show that the results hold at $i$. By the induction hypothesis,

$$
\frac{i-r+1}{2} R^{2} Z_{i-1}-\frac{i-r-1}{2} Z_{i+1} R^{2}+\sum_{j=r}^{i-2}\left[\frac{1}{4}-\frac{1}{4}(-1)^{j-r+1}+\frac{j-r+1}{2}(-1)^{j-r}\right] \varepsilon_{j} R F_{i-1}^{(r)}=0 .
$$

Multiplying $(-1) R$ from the left,

$$
-\frac{i-r+1}{2} R^{3} Z_{i-1}+\frac{i-r-1}{2} R Z_{i+1} R^{2}-\sum_{j=r}^{i-2}\left[\frac{1}{4}-\frac{1}{4}(-1)^{j-r+1}+\frac{j-r+1}{2}(-1)^{j-r}\right] \varepsilon_{j} R^{2} F_{i-1}^{(r)}=0
$$


Replacing $i$ by $i-1$ in (3.44), and multiplying by $\frac{i-r+1}{2}$,

$$
\begin{aligned}
& \frac{i-r+1}{2} R^{3} Z_{i-1}+\frac{i-r+1}{2} R^{2} Z_{i} R-\frac{i-r+1}{2} R Z_{i+1} R^{2}-\frac{i-r+1}{2} Z_{i+2} R^{3} \\
& -\frac{i-r+1}{2} \varepsilon_{i-1} R^{2} F_{i-1}^{(r)}=0 .
\end{aligned}
$$

Adding (3.50) and (3.51), and then removing $R$, we can get (3.45).

Lemma 3.12. For $r \leq i \leq d-r-2$,

$$
\begin{aligned}
& \frac{d-r-i}{2} R^{2} Z_{i}+R Z_{i+1} R-\frac{d-r-i}{2} Z_{i+2} R^{2} \\
& -\sum_{j=i}^{d-r-2}\left[\frac{1}{4}-\frac{1}{4}(-1)^{d-r-j}+\frac{d-r-j}{2}(-1)^{d-r-j}\right] \varepsilon_{j} R F_{i}^{(r)}=0, \quad \text { if } d-r-i \text { is even; } \\
& \frac{d-r-i-1}{2} R^{2} Z_{i}-\frac{d-r-i+1}{2} Z_{i+2} R^{2} \\
& +\sum_{j=i}^{d-r-2}\left[\frac{1}{4}-\frac{1}{4}(-1)^{d-r-j}+\frac{d-r-j}{2}(-1)^{d-r-j}\right] \varepsilon_{j} R F_{i}^{(r)}=0, \quad \text { if } d-r-i \text { is odd. }
\end{aligned}
$$

Proof. We show that the results hold using induction on $i$. Setting $i=d-r-2$ in (3.44), applying Lemma 3.9 and then removing $R$, we have

$$
R^{2} Z_{d-r-2}+R Z_{d-r-1} R-Z_{d-r} R^{2}-\varepsilon_{d-r-2} R F_{d-r-2}^{(r)}=0 .
$$

Hence, (3.52) holds at $i=d-r-2$.

Now suppose the results hold at $i+1$, where $d-r-i-1$ is a non-negative even integer. We will show that the results hold at $i$. By the induction hypothesis,

$$
\begin{aligned}
& \frac{d-r-i-1}{2} R^{2} Z_{i+1}+R Z_{i+2} R-\frac{d-r-i-1}{2} Z_{i+3} R^{2} \\
& -\sum_{j=i+1}^{d-r-2}\left[\frac{1}{4}-\frac{1}{4}(-1)^{d-r-j}+\frac{d-r-j}{2}(-1)^{d-r-j}\right] \varepsilon_{j} R F_{i+1}^{(r)}=0 .
\end{aligned}
$$

Multiplying (3.44) by $\frac{d-r-i-1}{2}$,

$$
\begin{aligned}
& \frac{d-r-i-1}{2} R^{3} Z_{i}+\frac{d-r-i-1}{2} R^{2} Z_{i+1} R-\frac{d-r-i-1}{2} R Z_{i+2} R^{2}-\frac{d-r-i-1}{2} Z_{i+3} R^{3} \\
& -\frac{d-r-i-1}{2} \varepsilon_{i} R^{2} F_{i}^{(r)}=0 .
\end{aligned}
$$

Multiplying (3.55) by $(-1) R$ from the right, adding (3.56), and then removing $R$, we can get (3.53).

Next, we suppose the results hold at $i+1$, where $d-r-i-1$ is a non-negative odd integer. We will show that the results hold at $i$. By the induction hypothesis,

$$
\frac{d-r-i-2}{2} R^{2} Z_{i+1}-\frac{d-r-i}{2} Z_{i+3} R^{2}+\sum_{j=i+1}^{d-r-2}\left[\frac{1}{4}-\frac{1}{4}(-1)^{d-r-j}+\frac{d-r-j}{2}(-1)^{d-r-j}\right] \varepsilon_{j} R F_{i+1}^{(r)}=0 .
$$


Multiplying (3.44) by $\frac{d-r-i}{2}$,

$$
\frac{d-r-i}{2} R^{3} Z_{i}+\frac{d-r-i}{2} R^{2} Z_{i+1} R-\frac{d-r-i}{2} R Z_{i+2} R^{2}-\frac{d-r-i}{2} Z_{i+3} R^{3}-\frac{d-r-i}{2} \varepsilon_{i} R^{2} F_{i}^{(r)}=0
$$

Multiplying (3.57) by $(-1) R$ from the right, adding (3.58), and then removing $R$, we can get (3.52).

LEMma 3.13. For $r+1 \leq i \leq d-r-1$,

(i) If $i-r$ and $d-r-i$ are odd, then

$$
(i-r+1) R Z_{i}-(d-r-i+1) Z_{i+1} R-e_{i}^{1} F_{i}^{(r)}=0,
$$

where

$$
\begin{aligned}
e_{i}^{1}= & (d-r-i+1) \sum_{j=r}^{i-1}\left[\frac{1}{4}-\frac{1}{4}(-1)^{j-r+1}+\frac{j-r+1}{2}(-1)^{j-r}\right] \varepsilon_{j} \\
& +(i-r+1) \sum_{j=i}^{d-r-2}\left[\frac{1}{4}-\frac{1}{4}(-1)^{d-r-j}+\frac{d-r-j}{2}(-1)^{d-r-j}\right] \varepsilon_{j} .
\end{aligned}
$$

(ii) If $i-r$ is even, $d-r-i$ is odd, then

$$
\begin{aligned}
& \frac{d-2 r+1}{2} R^{2} Z_{i}-\frac{d-2 r+1}{2} Z_{i+2} R^{2}+e_{i}^{2} R F_{i}^{(r)}=0, \\
& (d-2 r+1) R Z_{i}+e_{i}^{2^{\prime}} F_{i}^{(r)}=0,
\end{aligned}
$$

where

$$
\begin{aligned}
e_{i}^{2}= & \sum_{j=r}^{i-1}\left[\frac{1}{4}-\frac{1}{4}(-1)^{j-r+1}+\frac{j-r+1}{2}(-1)^{j-r}\right] \varepsilon_{j} \\
& +\sum_{j=i}^{d-r-2}\left[\frac{1}{4}-\frac{1}{4}(-1)^{d-r-j}+\frac{d-r-j}{2}(-1)^{d-r-j}\right] \varepsilon_{j}, \\
e_{i}^{2^{\prime}}= & (d-r-i+1) \sum_{j=r}^{i-1}\left[\frac{1}{4}-\frac{1}{4}(-1)^{j-r+1}+\frac{j-r+1}{2}(-1)^{j-r}\right] \varepsilon_{j} \\
& -(i-r) \sum_{j=i}^{d-r-2}\left[\frac{1}{4}-\frac{1}{4}(-1)^{d-r-j}+\frac{d-r-j}{2}(-1)^{d-r-j}\right] \varepsilon_{j} .
\end{aligned}
$$

(iii) If $i-r$ is odd, $d-r-i$ is even, then

$$
\begin{aligned}
& \frac{d-2 r+1}{2} R^{2} Z_{i}-\frac{d-2 r+1}{2} Z_{i+2} R^{2}-e_{i}^{3} R F_{i}^{(r)}=0, \\
& (d-2 r+1) Z_{i+1} R+e_{i}^{3^{\prime}} F_{i}^{(r)}=0,
\end{aligned}
$$


where

$$
\begin{aligned}
e_{i}^{3}= & \sum_{j=r}^{i-1}\left[\frac{1}{4}-\frac{1}{4}(-1)^{j-r+1}+\frac{j-r+1}{2}(-1)^{j-r}\right] \varepsilon_{j} \\
& +\sum_{j=i}^{d-r-2}\left[\frac{1}{4}-\frac{1}{4}(-1)^{d-r-j}+\frac{d-r-j}{2}(-1)^{d-r-j}\right] \varepsilon_{j}, \\
e_{i}^{3^{\prime}}= & (d-r-i) \sum_{j=r}^{i-1}\left[\frac{1}{4}-\frac{1}{4}(-1)^{j-r+1}+\frac{j-r+1}{2}(-1)^{j-r}\right] \varepsilon_{j} \\
& -(i-r+1) \sum_{j=i}^{d-r-2}\left[\frac{1}{4}-\frac{1}{4}(-1)^{d-r-j}+\frac{d-r-j}{2}(-1)^{d-r-j}\right] \varepsilon_{j} .
\end{aligned}
$$

(iv) If $i-r$ and $d-r-i$ are even, then

$$
(d-r-i) R Z_{i}-(i-r) Z_{i+1} R+e_{i}^{4} F_{i}^{(r)}=0
$$

where

$$
\begin{aligned}
e_{i}^{4}= & (d-r-i) \sum_{j=r}^{i-1}\left[\frac{1}{4}-\frac{1}{4}(-1)^{j-r+1}+\frac{j-r+1}{2}(-1)^{j-r}\right] \varepsilon_{j} \\
& +(i-r) \sum_{j=i}^{d-r-2}\left[\frac{1}{4}-\frac{1}{4}(-1)^{d-r-j}+\frac{d-r-j}{2}(-1)^{d-r-j}\right] \varepsilon_{j} .
\end{aligned}
$$

Proof. (i) Setting $i=d-r-1$ in (3.45), applying Lemma 3.9 and then removing $R$, we get (3.59) holds at $i=d-r-1$.

Suppose $r+1 \leq i \leq d-r-2$. Eliminating the term of $Z_{i+2} R^{2}$ from (3.45) and (3.53), and then removing $R$, we get (3.59).

(ii) Setting $i=d-r-1$ in (3.46) and using Lemma 3.9, we can get (3.60) holds at $i=d-r-1$. Removing $R$, we get (3.61) holds at $i=d-r-1$.

Suppose $r+1 \leq i \leq d-r-2$. Adding (3.46) and (3.53), we get (3.60). Eliminating the term of $Z_{i+2} R^{2}$ from (3.46) and (3.53), and then removing $R$, we get (3.61).

(iii) Eliminating the term of $R Z_{i+1} R$ from (3.45) and (3.52), we get (3.62). Similarly, eliminating the terms of $R^{2} Z_{i}, Z_{i+2} R^{2}$ from (3.45) and (3.52), and then removing $R$, we get (3.63).

(iv) Eliminating the term of $Z_{i+2} R^{2}$ from (3.46) and (3.52), and then removing $R$, we get (3.64).

LEMMA 3.14. With reference to Lemma 2.16, for $0 \leq i \leq d-2$,

$$
\varepsilon_{i}=4\left(b c^{*}+b^{*} c+2(i+1) c c^{*}\right) .
$$

Proof. Immediate from (2.7) by a conventional calculation. 
LEMmA 3.15. For $r+1 \leq i \leq d-r-1$, the scalars $e_{i}^{1}, e_{i}^{2}, e_{i}^{2^{\prime}}, e_{i}^{3}, e_{i}^{3^{\prime}}, e_{i}^{4}$ by Lemma 3.13 are given by

$$
\begin{aligned}
e_{i}^{1} & =(d-r-i+1)(i-r+1)\left(2 b c^{*}+2 b^{*} c+(d+2 i) c c^{*}\right), \\
e_{i}^{2} & =(d-2 r+1)(d-2 i-1) c c^{*}, \\
e^{2^{\prime}} & =-(d-r-i+1)(i-r)(d-2 r+1) c c^{*}, \\
e_{i}^{3} & =(d-2 r+1)\left(2 b c^{*}+2 b^{*} c+(d+2 i+1) c c^{*}\right), \\
e_{i}^{3^{\prime}} & =-(d-r-i)(i-r+1)(d-2 r+1) c c^{*}, \\
e_{i}^{4} & =(d-r-i)(i-r)\left(2 b c^{*}+2 b^{*} c+(d+2 i) c c^{*}\right) .
\end{aligned}
$$

Proof. Immediate from Lemmas 3.13 and 3.14 by a conventional calculation.

THEOREM 3.16. For $0 \leq r \leq h$ and $r+1 \leq i \leq d-r-1$,

(i) If $i-r$ and $d-r-i$ are odd, then

$$
\left(R L^{(0)}-\frac{d-r-i+1}{i-r+1} L^{(0)} R-(d-r-i+1) \mu_{i} I\right) U_{i}^{(r)}=0 .
$$

(ii) If $i-r$ is even, $d-r-i$ is odd, then

$$
\begin{aligned}
& \left(R^{2} L^{(0)}-L^{(0)} R^{2}+2(d-2 i-1) c c^{*} R\right) U_{i}^{(r)}=0, \\
& \left(R L^{(0)}-(d-r-i+1)(i-r) c c^{*} I\right) U_{i}^{(r)}=0 .
\end{aligned}
$$

(iii) If $i-r$ is odd, $d-r-i$ is even, then

$$
\begin{aligned}
& \left(R^{2} L^{(0)}-L^{(0)} R^{2}-2 \gamma_{i} R\right) U_{i}^{(r)}=0, \\
& \left(L^{(0)} R-(d-r-i)(i-r+1) c c^{*} I\right) U_{i}^{(r)}=0 .
\end{aligned}
$$

(iv) If $i-r$ and $d-r-i$ are even, then

$$
\left(R L^{(0)}-\frac{i-r}{d-r-i} L^{(0)} R+(i-r) \mu_{i} I\right) U_{i}^{(r)}=0,
$$

where

$$
\begin{aligned}
& \mu_{i}=2 b c^{*}+2 b^{*} c+(d+2 i) c c^{*}, \\
& \gamma_{i}=2 b c^{*}+2 b^{*} c+(d+2 i+1) c c^{*} .
\end{aligned}
$$

Proof. Immediate from Lemma 3.15 and equations (3.59)-(3.64).

To end this section, we give the following lemma for the future use.

LEMMA 3.17. Let $r$ denote an integer with $0 \leq r \leq h$. Let $Y$ denote a subspace of $U_{r}^{(r)}$ such that $L^{(0)} R Y \subseteq Y$. We set $W=\sum_{i=0}^{d-2 r} R^{i} Y$. Then $L^{(0)} W \subseteq W$.

Proof. We show

$$
L^{(0)} R^{i} Y \subseteq R^{i-1} Y \quad(1 \leq i \leq d-2 r) .
$$


We divide our proof into two cases in term of the parity of diameter $d$.

First we consider the case that $d$ is odd. If $i$ is even, pick any vector $u \in Y \subseteq U_{r}^{(r)}$, and observe that $R^{i-1} u$ belongs to $U_{r+i-1}^{(r)}$ by Lemma 2.3. Since $r+i-1-r$ is odd, applying (3.69) to $R^{i-1} u$, we find

$$
L^{(0)} R^{i} u=i(d-2 r-i+1) c c^{*} R^{i-1} u .
$$

Hence, $L^{(0)} R^{i} u \in R^{i-1} Y$. If $i$ is odd, we show (3.71) holds using induction on $i$. Observe that (3.71) holds at $i=1$ by our assumption. Now suppose $3 \leq i \leq d-2 r$, and suppose (3.71) holds at $i-2$. We will show (3.71) holds at $i$. Since $R^{i-2} u \in U_{r+i-2}^{(r)}$, and $r+i-2-r$ is odd, applying (3.68) to $R^{i-2} u$, we obtain

$$
L^{(0)} R^{i} u=R^{2} L^{(0)} R^{i-2} u-2 \gamma_{r+i-2} R^{i-1} u .
$$

Note that $L^{(0)} R^{i-2} u \in R^{i-3} Y$ by induction, so $R^{2} L^{(0)} R^{i-2} u \in R^{i-1} Y$. Hence, $L^{(0)} R^{i} u \in R^{i-1} Y$.

Next, we consider the case that $d$ is even. We show (3.71) holds using induction on $i$. Observe that (3.71) holds at $i=1$ by our assumption. Now suppose $2 \leq i \leq d-2 r$, and suppose (3.71) holds at $i-1$. We will show (3.71) holds at $i$. If $i$ is even, applying (3.65) to $R^{i-1} u$, we find

$$
L^{(0)} R^{i} u=\frac{i}{d-2 r-i+2} R L^{(0)} R^{i-1} u-i \mu_{r+i-1} R^{i-1} u .
$$

If $i$ is odd, applying (3.70) to $R^{i-1} u$, we find

$$
L^{(0)} R^{i} u=\frac{d-2 r-i+1}{i-1} R L^{(0)} R^{i-1} u+(d-2 r-i+1) \mu_{r+i-1} R^{i-1} u .
$$

These imply that $L^{(0)} R^{i} u \in \operatorname{span}\left\{R L^{(0)} R^{i-1} u, R^{i-1} u\right\}$. By the induction hypothesis, we have $L^{(0)} R^{i-1} u \in$ $R^{i-2} Y$, so $R L^{(0)} R^{i-1} u \in R^{i-1} Y$. Hence, $L^{(0)} R^{i} Y \subseteq R^{i-1} Y$. We have shown (3.71). We also have $L^{(0)} Y \subseteq$ $L^{(0)} U_{r}^{(r)}=0$ by Lemma 2.12. Thus, $L^{(0)} W \subseteq W$.

4. The basis for $V$. In this section, we assume the height of $A, A^{*}$ is 1 . That is $\rho_{0}=\rho_{d}=1$, $\rho_{1}=\rho_{2}=\cdots=\rho_{d-1} \geq 2$. Note that by [4, Lemma 4.1], $V$ is decomposed as $V=\sum_{i=0}^{d} U_{i}^{(0)}+\sum_{i=1}^{d-1} U_{i}^{(1)}$ (direct sum). Now, we give the bases for $U_{i}^{(0)}(0 \leq i \leq d)$ and $U_{i}^{(1)}(1 \leq i \leq d-1)$.

Fix a nonzero vector $u_{0}$ in $U_{0}$, and set $u_{i}=R^{i} u_{0}(1 \leq i \leq d)$.

LeMma 4.1. For $0 \leq i \leq d,\left\{u_{i}\right\}$ is a basis of $U_{i}^{(0)}$.

Proof. Immediate from Lemma 2.3.

LEMma 4.2. We have

(i) $L^{(+)} u_{0}=0, L^{(+)} u_{1}=0$;

(ii) $L^{(+)} u_{2} \neq 0$.

Proof. (i) Immediate from Lemma 2.12.

(ii) Suppose $L^{(+)} u_{2}=0$. Applying Lemma 3.4 to $u_{i}$ for $2 \leq i \leq d-1$,

$$
\begin{aligned}
& L^{(+)} u_{i+1}=L^{(+)} R u_{i}=-R L^{(+)} u_{i}, \quad \text { if } i \text { is even; } \\
& L^{(+)} u_{i+1}=L^{(+)} R u_{i}=-\frac{i+1}{i-1} R L^{(+)} u_{i}, \quad \text { if } i \text { is odd. }
\end{aligned}
$$


Combining with (i), these imply $L^{(+)} u_{i}=0$ for $0 \leq i \leq d$. So $L^{(+)} U^{(0)}=0$. Using (2.5) and Lemma 2.11, we have

$$
L U^{(0)}=L^{(-)} U^{(0)}+L^{(0)} U^{(0)}+L^{(+)} U^{(0)}=L^{(0)} U^{(0)} \subseteq U^{(0)} .
$$

Hence, $U^{(0)}$ is invariant under $L$. Also we have $R U^{(0)} \subseteq U^{(0)}$ by Lemma 2.7 , and $F_{i} U^{(0)}=U_{i}^{(0)} \subseteq U^{(0)}(0 \leq$ $i \leq d)$. From which we have $U^{(0)}=V$ by Lemma 2.2, a contradiction.

We set $v_{1}=L^{(+)} u_{2}, v_{i}=R^{i-1} v_{1} \quad(2 \leq i \leq d-1)$.

Lemma 4.3. For $1 \leq i \leq d-1, v_{i} \neq 0$ and $v_{i}$ lies in $U_{i}^{(1)}$.

Proof. Immediate from Lemmas 2.3 and 4.2.

Lemma 4.4. Suppose $\rho_{1}=2$. Then $\left\{v_{i}\right\}$ is a basis of $U_{i}^{(1)}(1 \leq i \leq d-1)$.

Proof. Immediate from Lemmas 2.5, 4.1 and 4.3.

Lemma 4.5. For $1 \leq i \leq d-1$,

$$
L^{(+)} u_{i+1}=b_{i} v_{i}
$$

where

$$
\begin{aligned}
b_{i} & =\frac{i+1}{2}, \quad \text { if } i \text { is odd } \\
b_{i} & =-\frac{i}{2}, \quad \text { if } i \text { is even. }
\end{aligned}
$$

Proof. We show (4.72) holds using induction on $i$. Observe that (4.72) holds for $i=1$, so we assume $2 \leq i \leq d-1$. Now suppose (4.72) holds at $i-1$. We will show (4.72) holds at $i$. If $i$ is even, applying (3.29) to $u_{i}$, then

$$
L^{(+)} u_{i+1}=L^{(+)} R u_{i}=-R L^{(+)} u_{i} .
$$

By induction,

$$
R L^{(+)} u_{i}=R b_{i-1} v_{i-1}=b_{i-1} v_{i}
$$

Hence,

$$
L^{(+)} u_{i+1}=-b_{i-1} v_{i}=-\frac{i}{2} v_{i}=b_{i} v_{i} .
$$

If $i$ is odd, applying (3.30) to $u_{i}$, then

$$
L^{(+)} u_{i+1}=L^{(+)} R u_{i}=-\frac{i+1}{i-1} R L^{(+)} u_{i}
$$

So,

$$
L^{(+)} u_{i+1}=-\frac{i+1}{i-1} b_{i-1} v_{i}=\frac{i+1}{2} v_{i}=b_{i} v_{i}
$$

Lemma 4.6. Suppose $\rho_{1} \geq 3$. Then $L^{(0)} v_{2}$ and $v_{1}$ are linearly independent.

Proof. By way of contradiction, we assume $L^{(0)} v_{2}$ lies in the span $Y$ of $\left\{v_{1}\right\}$. We set $W=\sum_{i=0}^{d-2} R^{i} Y$. Observe that $U^{(0)}+W$ is invariant under $R$ and $F_{i}(0 \leq i \leq d)$. We show that $U^{(0)}+W$ is invariant under $L$. 
Since $h=1$, we have $W \subseteq U^{(1)}$ and $L^{(+)} U^{(1)}=0$. So $L^{(+)} W=0$. Hence, $L W \subseteq L^{(-)} W+L^{(0)} W \subseteq$ $U^{(0)}+L^{(0)} W$. We have $L^{(0)} R Y \subseteq Y$ from our assumption $L^{(0)} v_{2} \in Y$, and this implies $L^{(0)} W \subseteq W$ by Lemma 3.17. Observe that $L^{(+)} U^{(0)} \subseteq W$ by Lemma 4.5, so that $L U^{(0)} \subseteq L^{(-)} U^{(0)}+L^{(0)} U^{(0)}+L^{(+)} U^{(0)} \subseteq U^{(0)}+W$, since $L^{(-)} U^{(0)}=0$. So $U^{(0)}+W$ is invariant under $L$. Therefore, $U^{(0)}+W$ is invariant under $L$, $R$ and $F_{i}(0 \leq i \leq d)$. From which we have $W=V$ by Lemma 2.2. This contradicts our assumption $\rho_{1} \geq 3$.

When $\rho_{1} \geq 3$, we set

$$
w_{1}=L^{(0)} v_{2}, \quad w_{i}=R^{i-1} w_{1}(2 \leq i \leq d-1) .
$$

LEMmA 4.7. Suppose $\rho_{1} \geq 3$. Then $v_{i}$ and $w_{i}$ are linearly independent for $1 \leq i \leq d-1$.

Proof. Immediate from Lemmas 2.3 and 4.6.

LEMMA 4.8. Suppose $\rho_{1}=3$. Then $\left\{v_{i}, w_{i}\right\}$ is a basis of $U_{i}^{(1)}(1 \leq i \leq d-1)$.

Proof. Immediate from Lemmas 2.4 and 4.7.

5. The action of $L$. Recall that $\left\{u_{i}\right\}$ is a basis of $U_{i}^{(0)},\left\{v_{i}\right\}$ is a basis of $U_{i}^{(1)}$ if $\rho_{1}=2$, and $\left\{v_{i}, w_{i}\right\}$ is a basis of $U_{i}^{(1)}$ if $\rho_{1}=3$. In this section, we consider the action of $L$ on these bases.

By Lemma 4.1, we know that $L^{(0)} u_{i+1}$ is a scalar multiple of $u_{i}$. We set

$$
L^{(0)} u_{i+1}=a_{i} u_{i} \quad(0 \leq i \leq d-1)
$$

for some scalars $a_{0}, a_{1}, \ldots, a_{d-1}$.

Clearly, $L^{(-)} v_{i+1}$ is a scalar multiple of $u_{i}$ by Lemmas 4.1 and 4.3 . So, we set

$$
L^{(-)} v_{i+1}=e_{i} u_{i} \quad(0 \leq i \leq d-2)
$$

for some scalars $e_{0}, e_{1}, \ldots, e_{d-2}$.

When $\rho_{1}=2$, by Lemma 4.4 ,

$$
L^{(0)} v_{1}=0, \quad L^{(0)} v_{i+1}=c_{i} v_{i} \quad(1 \leq i \leq d-2)
$$

for some scalars $c_{1}, \ldots, c_{d-2}$.

When $\rho_{1} \geq 3$, by Lemma 4.1 , we set

$$
L^{(-)} w_{i+1}=f_{i} u_{i} \quad(0 \leq i \leq d-2)
$$

for some scalars $f_{0}, f_{1}, \ldots, f_{d-2}$.

Similarly, when $\rho_{1}=3$, by Lemma 4.8 ,

$$
L^{(0)} w_{i+1}=s_{i} v_{i}+t_{i} w_{i} \quad(1 \leq i \leq d-2)
$$

for some scalars $s_{i}, t_{i}$.

Now we determine the parameters $a_{i}, e_{i}, c_{i}, f_{i}, m_{i}, n_{i}, s_{i}, t_{i}$. We divide the arguments into two cases in term of the parity of diameter $d$.

Case $I: d$ is odd. By equations (5.75)-(5.79), we have the following lemmas. 
Lemma 5.1. For $0 \leq i \leq d-1$,

$$
\begin{aligned}
& a_{i}=(i+1)(d-i) c c^{*}, \quad \text { if } i \text { is odd } ; \\
& a_{i}=a_{0}-i\left(2 b c^{*}+2 b^{*} c+(d+i+1) c c^{*}\right), \quad \text { if } i \text { is even. }
\end{aligned}
$$

Proof. We first show (5.80) holds. Applying (3.69) to $u_{i}$,

$$
L^{(0)} R u_{i}=(i+1)(d-i) c c^{*} u_{i} .
$$

Note that $L^{(0)} R u_{i}=L^{(0)} u_{i+1}=a_{i} u_{i}$. Hence, $a_{i}=(i+1)(d-i) c c^{*}$.

Next, we show (5.81) holds using induction on $i$. Clearly (5.81) holds for $i=0$, so we assume $2 \leq i \leq d-1$. Now suppose (5.81) holds at $i-2$. We will show (5.81) holds at $i$. Applying (3.68) to $u_{i-1}$,

$$
L^{(0)} R^{2} u_{i-1}=R^{2} L^{(0)} u_{i-1}-2 \gamma_{i-1} R u_{i-1} .
$$

Note that $L^{(0)} R^{2} u_{i-1}=L^{(0)} u_{i+1}=a_{i} u_{i}$, and $R^{2} L^{(0)} u_{i-1}=R^{2} a_{i-2} u_{i-2}=a_{i-2} u_{i}$. So $a_{i}=a_{i-2}-2 \gamma_{i-1}$. By induction,

$$
a_{i-2}=a_{0}-(i-2)\left(2 b c^{*}+2 b^{*} c+(d+i-1) c c^{*}\right) .
$$

Hence, (5.81) holds.

Lemma 5.2. For $0 \leq i \leq d-2$,

$$
\begin{aligned}
& e_{i}=-\frac{d-i}{d-1} e_{0}, \quad \text { if } i \text { is odd; } \\
& e_{i}=\frac{d-i-1}{d-1} e_{0}, \quad \text { if } i \text { is even. }
\end{aligned}
$$

Proof. We show that the results hold using induction on $i$. Observe that the results hold at $i=0$. Now suppose $1 \leq i \leq d-2$, and suppose the results hold at $i-1$. We will show that the results hold at $i$. If $i$ is odd, applying (3.42) to $v_{i}$, then

$$
L^{(-)} R v_{i}=-R L^{(-)} v_{i}
$$

Note that $L^{(-)} R v_{i}=L^{(-)} v_{i+1}=e_{i} u_{i}$, and $R L^{(-)} v_{i}=R e_{i-1} u_{i-1}=e_{i-1} u_{i}$. So $e_{i}=-e_{i-1}$. By induction,

$$
e_{i-1}=\frac{d-i}{d-1} e_{0}
$$

Hence, (5.82) holds. If $i$ is even, applying (3.43) to $v_{i}$, then

$$
L^{(-)} R v_{i}=-\frac{d-i-1}{d-i+1} R L^{(-)} v_{i}
$$

So,

$$
e_{i}=-\frac{d-i-1}{d-i+1} e_{i-1}
$$

By induction,

$$
e_{i-1}=-\frac{d-i+1}{d-1} e_{0} .
$$

Hence, (5.83) holds. 
Lemma 5.3. Suppose $\rho_{1}=2$. Then, for $1 \leq i \leq d-2$,

$$
\begin{aligned}
& c_{i}=c_{1}-(i-1)\left(2 b c^{*}+2 b^{*} c+(d+i+2) c c^{*}\right), \quad \text { if } i \text { is odd; } \\
& c_{i}=i(d-i-1) c c^{*}, \quad \text { if } i \text { is even. }
\end{aligned}
$$

Proof. We first show (5.84) holds using induction on $i$. Observe that (5.84) holds for $i=1$, so we assume $3 \leq i \leq d-2$. Now suppose (5.84) holds at $i-2$. We will show (5.84) holds at $i$. Applying (3.68) to $v_{i-1}$,

$$
L^{(0)} R^{2} v_{i-1}=R^{2} L^{(0)} v_{i-1}-2 \gamma_{i-1} R v_{i-1} .
$$

Note that $L^{(0)} R^{2} v_{i-1}=L^{(0)} v_{i+1}=c_{i} v_{i}$, and $R^{2} L^{(0)} v_{i-1}=R^{2} c_{i-2} v_{i-2}=c_{i-2} v_{i}$. Thus, $c_{i}=c_{i-2}-2 \gamma_{i-1}$. By induction,

$$
c_{i-2}=c_{1}-(i-3)\left(2 b c^{*}+2 b^{*} c+(d+i) c c^{*}\right)
$$

Hence, (5.84) follows.

Next, we will show (5.85) holds. Applying (3.69) to $v_{i}$,

$$
L^{(0)} R v_{i}=i(d-i-1) c c^{*} v_{i}
$$

Note that $L^{(0)} R v_{i}=L^{(0)} v_{i+1}=c_{i} v_{i}$. Hence, (5.85) holds.

Lemma 5.4. Suppose $\rho_{1} \geq 3$. Then, for $0 \leq i \leq d-2$,

$$
\begin{aligned}
& f_{i}=-\frac{d-i}{d-1} f_{0}, \quad \text { if } i \text { is odd; } \\
& f_{i}=\frac{d-i-1}{d-1} f_{0}, \quad \text { if } i \text { is even. }
\end{aligned}
$$

Proof. Similar to the proof of Lemma 5.2.

Lemma 5.5. Suppose $\rho_{1} \geq 3$. Then

$$
L^{(0)} v_{i+1}=m_{i} v_{i}+n_{i} w_{i} \quad(1 \leq i \leq d-2),
$$

where

$$
\begin{aligned}
& m_{i}=-(i-1)\left(2 b c^{*}+2 b^{*} c+(d+i+2) c c^{*}\right), n_{i}=1, \quad \text { if } i \text { is odd } \\
& m_{i}=i(d-i-1) c c^{*}, n_{i}=0, \quad \text { if } i \text { is even. }
\end{aligned}
$$

Proof. We divide our proof into two cases in term of the parity of $i$. First suppose $i$ is odd. We will show (5.88) holds using induction on $i$. Observe that (5.88) holds for $i=1$ with $m_{1}=0, n_{1}=1$, since $L^{(0)} v_{2}=w_{1}$. Now we assume $3 \leq i \leq d-2$, and suppose (5.88) holds at $i-2$. We will show (5.88) holds at i. Applying (3.68) to $v_{i-1}$,

$$
L^{(0)} R^{2} v_{i-1}=R^{2} L^{(0)} v_{i-1}-2 \gamma_{i-1} R v_{i-1} \text {. }
$$

Note that $L^{(0)} R^{2} v_{i-1}=L^{(0)} v_{i+1}$, and $R v_{i-1}=v_{i}$. By induction, we have

$$
R^{2} L^{(0)} v_{i-1}=R^{2}\left(m_{i-2} v_{i-2}+n_{i-2} w_{i-2}\right)=m_{i-2} v_{i}+n_{i-2} w_{i}
$$

Hence,

$$
\begin{aligned}
L^{(0)} v_{i+1} & =\left(-(i-3)\left(2 b c^{*}+2 b^{*} c+(d+i) c c^{*}\right)-2\left(2 b c^{*}+2 b^{*} c+(d+2 i-1) c c^{*}\right)\right) v_{i}+w_{i} \\
& =-(i-1)\left(2 b c^{*}+2 b^{*} c+(d+i+2) c c^{*}\right) v_{i}+w_{i}
\end{aligned}
$$


Next suppose $i$ is even. Applying (3.67) to $v_{i+1}$ to get

$$
R L^{(0)} v_{i+1}=i(d-i-1) c c^{*} v_{i+1} .
$$

Removing $R$, we find

$$
L^{(0)} v_{i+1}=i(d-i-1) c c^{*} v_{i}=m_{i} v_{i}+n_{i} w_{i} .
$$

Lemma 5.6. Suppose $\rho_{1}=3$. Then, for $1 \leq i \leq d-2$,

$$
\begin{aligned}
& s_{i}=s_{1}, t_{i}=t_{1}-(i-1)\left(2 b c^{*}+2 b^{*} c+(d+i+2) c c^{*}\right), \quad \text { if } i \text { is odd } \\
& s_{i}=0, t_{i}=i(d-i-1) c c^{*}, \quad \text { if } i \text { is even. }
\end{aligned}
$$

Proof. Similar to the proof of Lemma 5.5.

LEMma 5.7. When $\rho_{1} \geq 3$, the following hold with the values of $a_{i}, b_{i}, e_{i}, f_{i}, m_{i}, n_{i}$ given by (5.80), (5.81), (4.73), (4.74), (5.82), (5.83), (5.86), (5.87), (5.89) and (5.90).

(i) $L u_{0}=0, L u_{1}=a_{0} u_{0}, L u_{i+1}=a_{i} u_{i}+b_{i} v_{i}(1 \leq i \leq d-1)$,

(ii) $L v_{1}=e_{0} u_{0}, L v_{i+1}=e_{i} u_{i}+m_{i} v_{i}+n_{i} w_{i}(1 \leq i \leq d-2)$,

(iii) $L^{(-)} w_{i+1}=f_{i} u_{i}(0 \leq i \leq d-2)$.

Proof. Immediate from Lemma 2.11, and equations (4.72), (5.75), (5.76), (5.78) and (5.88).

Case II: $d$ is even. Similarly, by equations (5.75)-(5.79), we have the following lemmas.

LEMma 5.8. For $0 \leq i \leq d-1$,

$$
\begin{aligned}
& a_{i}=(i+1)\left(\frac{a_{0}}{d}+\sum_{k=1}^{i}(-1)^{k} \mu_{k}\right), \quad \text { if } i \text { is odd; } \\
& a_{i}=(d-i)\left(\frac{a_{0}}{d}+\sum_{k=1}^{i}(-1)^{k} \mu_{k}\right), \quad \text { if } i \text { is even. }
\end{aligned}
$$

Proof. We show that the results hold using induction on $i$. Observe that the results hold for $i=0$, so we assume $1 \leq i \leq d-1$, and suppose the results hold at $i-1$. We will show that the results hold at $i$. If $i$ is odd, applying (3.65) to $u_{i}$, then

$$
L^{(0)} R u_{i}=\frac{i+1}{d-i+1} R L^{(0)} u_{i}-(i+1) \mu_{i} u_{i} .
$$

Note that $L^{(0)} R u_{i}=L^{(0)} u_{i+1}=a_{i} u_{i}$, and $R L^{(0)} u_{i}=R\left(a_{i-1} u_{i-1}\right)=a_{i-1} R u_{i-1}=a_{i-1} u_{i}$. Hence,

$$
a_{i}=\frac{i+1}{d-i+1} a_{i-1}-(i+1) \mu_{i} .
$$

By induction,

$$
a_{i-1}=(d-i+1)\left(\frac{a_{0}}{d}+\sum_{k=1}^{i-1}(-1)^{k} \mu_{k}\right) .
$$

Now (5.93) holds. If $i$ is even, applying (3.70) to $u_{i}$, then

$$
L^{(0)} R u_{i}=\frac{d-i}{i} R L^{(0)} u_{i}+(d-i) \mu_{i} u_{i} .
$$


So

$$
a_{i}=\frac{d-i}{i} a_{i-1}+(d-i) \mu_{i}
$$

By induction,

$$
a_{i-1}=i\left(\frac{a_{0}}{d}+\sum_{k=1}^{i-1}(-1)^{k} \mu_{k}\right)
$$

Now (5.94) holds.

LEMMA 5.9. For $0 \leq i \leq d-2$,

$$
\begin{aligned}
& e_{i}=-\frac{d-i-1}{d} e_{0}, \quad \text { if } i \text { is odd; } \\
& e_{i}=\frac{d-i}{d} e_{0}, \quad \text { if } i \text { is even. }
\end{aligned}
$$

Proof. Similar to the proof of Lemma 5.2.

Lemma 5.10. Suppose $\rho_{1}=2$. Then, for $1 \leq i \leq d-2$,

$$
\begin{aligned}
& c_{i}=(d-i-1)\left(\frac{c_{1}}{d-2}-\sum_{k=2}^{i}(-1)^{k} \mu_{k}\right), \quad \text { if } i \text { is odd } \\
& c_{i}=i\left(\frac{c_{1}}{d-2}-\sum_{k=2}^{i}(-1)^{k} \mu_{k}\right), \quad \text { if } i \text { is even. }
\end{aligned}
$$

Proof. We show that the results hold using induction on $i$. Observe that the results hold for $i=1$. So we assume $2 \leq i \leq d-2$. Now suppose the results hold at $i-1$. We will show that the results hold at $i$. If $i$ is odd, applying (3.70) to $v_{i}$, then

$$
L^{(0)} R v_{i}=\frac{d-i-1}{i-1} R L^{(0)} v_{i}+(d-i-1) \mu_{i} v_{i}
$$

Note that $L^{(0)} R v_{i}=L^{(0)} v_{i+1}=c_{i} v_{i}$, and $R L^{(0)} v_{i}=R c_{i-1} v_{i-1}=c_{i-1} v_{i}$. Hence,

$$
c_{i}=\frac{d-i-1}{i-1} c_{i-1}+(d-i-1) \mu_{i} .
$$

By induction,

$$
c_{i-1}=(i-1)\left(\frac{c_{1}}{d-2}-\sum_{k=2}^{i-1}(-1)^{k} \mu_{k}\right) .
$$

Now (5.97) holds. If $i$ is even, applying (3.65) to $v_{i}$, then

$$
L^{(0)} R v_{i}=\frac{i}{d-i} R L^{(0)} v_{i}-i \mu_{i} v_{i}
$$

So

$$
c_{i}=\frac{i}{d-i} c_{i-1}-i \mu_{i}
$$

By induction,

$$
c_{i-1}=(d-i)\left(\frac{c_{1}}{d-2}-\sum_{k=2}^{i-1}(-1)^{k} \mu_{k}\right) .
$$

Now (5.98) holds. 
Lemma 5.11. Suppose $\rho_{1} \geq 3$. Then, for $0 \leq i \leq d-2$,

$$
\begin{aligned}
& f_{i}=-\frac{d-i-1}{d} f_{0}, \quad \text { if } i \text { is odd; } \\
& f_{i}=\frac{d-i}{d} f_{0}, \quad \text { if } i \text { is even. }
\end{aligned}
$$

Proof. Similar to the proof of Lemma 5.2.

Lemma 5.12. Suppose $\rho_{1} \geq 3$. Then

$$
L^{(0)} v_{i+1}=m_{i} v_{i}+n_{i} w_{i} \quad(1 \leq i \leq d-2),
$$

where

$$
\begin{aligned}
& m_{i}=-(d-i-1) \sum_{k=2}^{i}(-1)^{k} \mu_{k}, n_{i}=\frac{d-i-1}{d-2}, \quad \text { if } i \text { is odd } \\
& m_{i}=-i \sum_{k=2}^{i}(-1)^{k} \mu_{k}, n_{i}=\frac{i}{d-2}, \quad \text { if } i \text { is even. }
\end{aligned}
$$

Proof. We show (5.101) holds using induction on $i$. Obverse that (5.101) holds for $i=1$ with $m_{1}=$ $0, n_{1}=1$, since $L^{(0)} v_{2}=w_{1}$. Now we assume $2 \leq i \leq d-2$, and suppose (5.101) holds at $i-1$. We will show (5.101) holds at $i$. If $i$ is odd, applying (3.70) to $v_{i}$, then

$$
L^{(0)} R v_{i}=\frac{d-i-1}{i-1} R L^{(0)} v_{i}+(d-i-1) \mu_{i} v_{i} .
$$

Note that $L^{(0)} R v_{i}=L^{(0)} v_{i+1}$. By induction,

$$
R L^{(0)} v_{i}=R\left(m_{i-1} v_{i-1}+n_{i-1} w_{i-1}\right)=m_{i-1} v_{i}+n_{i-1} w_{i} .
$$

Hence,

$$
\begin{aligned}
L^{(0)} v_{i+1} & =\frac{d-i-1}{i-1}\left(-(i-1) \sum_{k=2}^{i-1}(-1)^{k} \mu_{k} v_{i}+\frac{i-1}{d-2} w_{i}\right)+(d-i-1) \mu_{i} v_{i} \\
& =-(d-i-1) \sum_{k=2}^{i}(-1)^{k} \mu_{k} v_{i}+\frac{d-i-1}{d-2} w_{i} .
\end{aligned}
$$

If $i$ is even, applying (3.65) to $v_{i}$, then

$$
L^{(0)} R v_{i}=\frac{i}{d-i} R L^{(0)} v_{i}-i \mu_{i} v_{i} .
$$

Hence

$$
\begin{aligned}
L^{(0)} v_{i+1} & =\frac{i}{d-i}\left(-(d-i) \sum_{k=2}^{i-1}(-1)^{k} \mu_{k} v_{i}+\frac{d-i}{d-2} w_{i}\right)-i \mu_{i} v_{i} \\
& =-i \sum_{k=2}^{i}(-1)^{k} \mu_{k} v_{i}+\frac{i}{d-2} w_{i}
\end{aligned}
$$


LEMma 5.13. Suppose $\rho_{1}=3$. Then, for $1 \leq i \leq d-2$,

$$
\begin{aligned}
& s_{i}=\frac{d-i-1}{d-2} s_{1}, \quad t_{i}=(d-i-1)\left(\frac{t_{1}}{d-2}-\sum_{k=2}^{i}(-1)^{k} \mu_{k}\right), \quad \text { if } i \text { is odd; } \\
& s_{i}=\frac{i}{d-2} s_{1}, \quad t_{i}=i\left(\frac{t_{1}}{d-2}-\sum_{k=2}^{i}(-1)^{k} \mu_{k}\right), \quad \text { if } i \text { is even. }
\end{aligned}
$$

Proof. Similar to the proof of Lemma 5.12 .

LEMMA 5.14. When $\rho_{1} \geq 3$, the following hold with the values of $a_{i}, b_{i}, e_{i}, f_{i}, m_{i}, n_{i}$ given by (5.93), (5.94), (4.73), (4.74), (5.95), (5.96), (5.99), (5.100), (5.102) and (5.103).

(i) $L u_{0}=0, L u_{1}=a_{0} u_{0}, L u_{i+1}=a_{i} u_{i}+b_{i} v_{i}(1 \leq i \leq d-1)$,

(ii) $L v_{1}=e_{0} u_{0}, L v_{i+1}=e_{i} u_{i}+m_{i} v_{i}+n_{i} w_{i}(1 \leq i \leq d-2)$,

(iii) $L^{(-)} w_{i+1}=f_{i} u_{i}(0 \leq i \leq d-2)$.

Proof. Similar to the proof of Lemma 5.7.

6. Determining the shape of the tridiagonal pair. Recall that $u_{0} \in U_{0}, u_{i}=R^{i} u_{0}(1 \leq i \leq d)$, $v_{1}=L^{(+)} u_{2}, v_{i}=R^{i-1} v_{2}(2 \leq i \leq d-1), w_{1}=L^{(0)} v_{2}$, and $w_{i}=R^{i-1} w_{1}(2 \leq i \leq d-1)$. In this section, we assume the height of $A, A^{*}$ is 1 , and determine the shape of $A, A^{*}$.

Lemma 6.1. Suppose $\rho_{1} \geq 4$. Then the vectors $v_{1}, w_{1}, L^{(0)} w_{2}$ are linearly independent.

Proof. By way of contradiction, we suppose $v_{1}, w_{1}, L^{(0)} w_{2}$ are linearly dependent. Since $v_{1}, w_{1}$ are linearly independent by Lemma 4.7 and by our assumption, $L^{(0)} w_{2}$ lies in $Y=\operatorname{span}\left\{v_{1}, w_{1}\right\}$. We $\operatorname{set}$ $W=\sum_{i=0}^{d-2} R^{i} Y$. Observe that $U^{(0)}+W$ is invariant under $R$ and $F_{i}(0 \leq i \leq d)$. We show $U^{(0)}+W$ is invariant under $L$.

Obverse that $R Y=\operatorname{span}\left\{v_{2}, w_{2}\right\}$ and $L^{(0)} v_{2}=w_{1} \in Y$, so that $L^{(0)} R Y \subseteq Y$. Hence, $W$ is invariant under $L^{(0)}$ by Lemma 3.17. This implies $L W \subseteq L^{(-)} W+L^{(0)} W \subseteq U^{(0)}+W$. Moreover, $L^{(+)} U^{(0)} \subseteq W$ by Lemma 4.5. So, $U^{(0)}+W$ is invariant under $L$. Therefore, $U^{(0)}+W$ is invariant under $L, R$ and $F_{i}(0 \leq i \leq d)$. From which we have $U^{(0)}+W=V$ by Lemma 2.2. It follows that $U_{1}=\left\{u_{1}, v_{1}, w_{1}\right\}$, contradicting our assumption $\rho_{1} \geq 4$.

Lemma 6.2. Suppose $\rho_{1} \geq 4$, and we set $L^{(0)} w_{2}=x_{1}$. Then

(i) If $d=3$, we have

$$
\begin{aligned}
L^{3} R u_{3} & =0 \\
L^{2} R L u_{3} & =\left(a_{1} a_{2} a_{2}+e_{1} a_{2} b_{2}\right) u_{1}+a_{2} a_{2} v_{1}+a_{2} b_{2} w_{1}, \\
L R L^{2} u_{3} & =\left(a_{1}\left(a_{1} a_{2}+e_{1} b_{2}\right)+e_{1} a_{2}+f_{1} b_{2}\right) u_{1}+\left(a_{1} a_{2}+e_{1} b_{2}\right) v_{1}+a_{2} w_{1}+b_{2} x_{1}, \\
R L^{3} u_{3} & \in \operatorname{span}\left\{u_{1}\right\} \\
L^{2} u_{3} & =\left(a_{1} a_{2}+e_{1} b_{2}\right) u_{1}+a_{2} v_{1}+b_{2} w_{1} .
\end{aligned}
$$


(ii) If $d \geq 4$, we have

$$
\begin{aligned}
L^{3} R u_{3} & =\left(a_{2} a_{3}+e_{2} b_{3}\right)\left(a_{1} u_{1}+v_{1}\right)+\left(b_{2} a_{3}+m_{2} b_{3}\right)\left(e_{1} u_{1}+w_{1}\right)+n_{2} b_{3}\left(f_{1} u_{1}+x_{1}\right), \\
L^{2} R L u_{3} & =\left(a_{2} a_{2}+b_{2} e_{2}\right)\left(a_{1} u_{1}+v_{1}\right)+\left(a_{2} b_{2}+b_{2} m_{2}\right)\left(e_{1} u_{1}+w_{1}\right)+b_{2} n_{2}\left(f_{1} u_{1}+x_{1}\right), \\
L R L^{2} u_{3} & =\left(a_{1}\left(a_{1} a_{2}+e_{1} b_{2}\right)+e_{1} a_{2}+f_{1} b_{2}\right) u_{1}+\left(a_{1} a_{2}+e_{1} b_{2}\right) v_{1}+a_{2} w_{1}+b_{2} x_{1}, \\
R L^{3} u_{3} & \in \operatorname{span}\left\{u_{1}\right\}, \\
L^{2} u_{3} & =\left(a_{1} a_{2}+e_{1} b_{2}\right) u_{1}+a_{2} v_{1}+b_{2} w_{1} .
\end{aligned}
$$

Proof. Immediate from Lemmas 2.3, 2.11, 5.7 and 5.14, and (5.78) by a routine computation.

LEMMA 6.3. $\rho_{1} \leq 3$.

Proof. By way of contradiction, we suppose $\rho_{1} \geq 4$. Observe that $u_{1}, v_{1}, w_{1}, x_{1}$ are linearly independent by Lemmas 2.6 and 6.1. Applying (2.6) to $u_{3}$,

$$
L^{3} R u_{3}+L^{2} R L u_{3}-L R L^{2} u_{3}-R L^{3} u_{3}+\varepsilon_{1} L^{2} u_{3}=0 .
$$

According to the parity of diameter $d$ and the range of diameter $d$, we divide our computation into three cases: (I) $d=3$; (II) $d$ is odd and $d \geq 5$; (III) $d$ is even.

Case (I) $d=3$.

Obverse that the coefficient of $x_{1}$ in (6.106) becomes $-b_{2}$ by Lemma 6.2 , so that $-b_{2}=0$, contradicting our convention.

Case (II) $d$ is odd and $d \geq 4$.

Looking at the coefficients of $x_{1}$ in (6.106), we have $n_{2} b_{3}+b_{2} n_{2}-b_{2}=0$ by Lemma 6.2 , so that $1=0$, contradicting our convention.

Case (III) $d$ is even.

Looking at the coefficients of $x_{1}$ in (6.106) by Lemma 6.2 ,

$$
\frac{2}{d-2} \cdot \frac{3+1}{2}+(-1) \cdot \frac{2}{d-2}-(-1)=\frac{d}{d-2}=0,
$$

so that $d=0$, contradicting to the assumption. These complete the proof of $\rho_{1} \leq 3$.

THEOREM 6.4. One of the following holds.

(i) $\rho_{0}=1, \rho_{1}=\rho_{2}=\cdots=\rho_{d-1}=2, \rho_{d}=1$,

(ii) $d=3, \rho_{0}=1, \rho_{1}=\rho_{2}=3, \rho_{3}=1$.

Proof. Since $\mathbb{K}$ is an algebraically closed field, by [7, Theorem 1.3], $\rho_{0}=1$. And by Lemma $6.3, \rho_{1} \leq 3$. So, $\rho_{1}=2$ or $\rho_{1}=3$, since $h=1$. If $\rho_{1}=2$, then (i) holds. If $\rho_{1}=3$, we need to show $d=3$. Applying (2.6) to $v_{3}$,

$$
L^{3} R v_{3}+L^{2} R L v_{3}-L R L^{2} v_{3}-R L^{3} v_{3}+\varepsilon_{1} L^{2} v_{3}=0 .
$$

From Lemmas 2.11, 3.14, 5.7 and 5.14, we can compute each term of (6.107). The term of $L^{3} R v_{3}$ vanishes 
when $d=4$. When $d \geq 5$, it becomes

$$
\begin{aligned}
L^{3} R v_{3}= & \left(a_{1}\left(a_{2} e_{3}+e_{2} m_{3}+f_{2} n_{3}\right)+e_{1}\left(b_{2} e_{3}+m_{2} m_{3}+s_{2} n_{3}\right)+f_{1}\left(n_{2} m_{3}+t_{2} n_{3}\right)\right) u_{1} \\
& +\left(\left(a_{2} e_{3}+e_{2} m_{3}+f_{2} n_{3}\right)+s_{1}\left(n_{2} m_{3}+t_{2} n_{3}\right)\right) v_{1} \\
& +\left(\left(b_{2} e_{3}+m_{2} m_{3}+s_{2} n_{3}\right)+t_{1}\left(n_{2} m_{3}+t_{2} n_{3}\right)\right) w_{1}, \\
L^{2} R L v_{3}= & \left(a_{1}\left(a_{2} e_{2}+e_{2} m_{2}+f_{2} n_{2}\right)+e_{1}\left(b_{2} e_{2}+m_{2} m_{2}+n_{2} s_{2}\right)+f_{1}\left(m_{2} n_{2}+n_{2} t_{2}\right)\right) u_{1} \\
& +\left(\left(a_{2} e_{2}+e_{2} m_{2}+f_{2} n_{2}\right)+s_{1}\left(m_{2} n_{2}+n_{2} t_{2}\right)\right) v_{1} \\
& +\left(\left(b_{2} e_{2}+m_{2} m_{2}+n_{2} s_{2}\right)+t_{1}\left(m_{2} n_{2}+n_{2} t_{2}\right)\right) w_{1}, \\
L R L^{2} v_{3}= & \left(a_{1}\left(a_{1} e_{2}+e_{1} m_{2}+f_{1} n_{2}\right)+e_{1}\left(e_{2}+s_{1} n_{2}\right)+f_{1}\left(m_{2}+n_{2} t_{2}\right)\right) u_{1} \\
& +\left(\left(a_{1} e_{2}+e_{1} m_{2}+f_{1} n_{2}\right)+s_{1}\left(m_{2}+t_{1} n_{2}\right)\right) v_{1}+\left(\left(e_{2}+s_{1} n_{2}\right)+t_{1}\left(m_{2}+t_{1} n_{2}\right)\right) w_{1}, \\
R L^{3} v_{3} \in & \operatorname{span}\left\{u_{1}\right\} \\
L^{2} v_{3}= & \left(a_{1} e_{2}+e_{1} m_{2}+f_{1} n_{2}\right) u_{1}+\left(e_{2}+s_{1} n_{2}\right) v_{1}+\left(m_{2}+t_{1} n_{2}\right) w_{1} .
\end{aligned}
$$

According to the parity of diameter $d$ and the range of diameter $d$, we divide the arguments into four cases: (I) $d=3$; (II) $d$ is odd and $d \geq 5$; (III) $d=4$; (IV) $d$ is even and $d \geq 6$. To show (I) holds, we show that (II), (III), and (IV) do not occur.

Suppose case (II) holds for a contradiction.

By a routine computation, the coefficient of $w_{1}$ in (6.107) becomes

$$
\begin{aligned}
& b_{2} e_{3}+m_{2} m_{3}+s_{2} n_{3}+t_{1}\left(n_{2} m_{3}+t_{2} n_{3}\right)+b_{2} e_{2}+m_{2} m_{2}+n_{2} s_{2}+t_{1}\left(m_{2} n_{2}+n_{2} t_{2}\right) \\
& -\left(e_{2}+s_{1} n_{2}+t_{1}\left(m_{2}+t_{1} n_{2}\right)\right)+\varepsilon_{1}\left(m_{2}+t_{1} n_{2}\right)=-\frac{d-3}{d-1} e_{0}=0 .
\end{aligned}
$$

It follows that $e_{0}=0$. And this implies $e_{i}=0(0 \leq i \leq d-2)$. The coefficient of $v_{1}$ becomes

$$
\begin{aligned}
& f_{2} n_{3}+s_{1}\left(n_{2} m_{3}+t_{2} n_{3}\right)+f_{2} n_{2}+s_{1}\left(m_{2} n_{2}+n_{2} t_{2}\right)-\left(f_{1} n_{2}+s_{1}\left(m_{2}+t_{1} n_{2}\right)\right)+\varepsilon_{1} s_{1} n_{2} \\
& =\frac{d-3}{d-1} f_{0}=0 .
\end{aligned}
$$

It follows that $f_{0}=0$. And this implies $f_{i}=0(0 \leq i \leq d-2)$. Since $L^{(-)} w_{i+1}=f_{i} u_{i}, L^{(-)} v_{i+1}=$ $e_{i} v_{i}(0 \leq i \leq d-2)$, we have $L^{(-)} U^{(1)}=0$ by Lemma 4.8. Hence, $L U^{(1)} \subseteq U^{(1)}$. Since $U^{(1)}$ is invariant under $R$ and $F_{i}(0 \leq i \leq d)$, we get $U^{(1)}=V$ by Lemma 2.2, a contradiction.

Suppose case (III) holds for a contradiction.

The coefficient of $w_{1}$ in (6.107) becomes

$$
b_{2} e_{2}+m_{2} m_{2}+n_{2} s_{2}+t_{1}\left(m_{2} n_{2}+n_{2} t_{2}\right)-\left(e_{2}+s_{1} n_{2}+t_{1}\left(m_{2}+t_{1} n_{2}\right)\right)+\varepsilon_{1}\left(m_{2}+t_{1} n_{2}\right)=-e_{0}=0,
$$

It implies $e_{i}=0(0 \leq i \leq d-2)$. The coefficient of $v_{1}$ becomes

$$
f_{2} n_{2}+s_{1}\left(m_{2} n_{2}+n_{2} t_{2}\right)-\left(f_{1} n_{2}+s_{1}\left(m_{2}+t_{1} n_{2}\right)\right)+\varepsilon_{1} s_{1} n_{2}=f_{0}=0,
$$

It implies $f_{i}=0(0 \leq i \leq d-2)$. Hence, $L U^{(1)} \subseteq U^{(1)}$. Since $U^{(1)}$ is invariant under $R$ and $F_{i}(0 \leq i \leq d)$, we get $U^{(1)}=V$ by Lemma 2.2, a contradiction. 
Suppose case (IV) holds for a contradiction.

By a routine computation, the coefficient of $w_{1}$ in (6.107) becomes

$$
\begin{aligned}
& b_{2} e_{3}+m_{2} m_{3}+s_{2} n_{3}+t_{1}\left(n_{2} m_{3}+t_{2} n_{3}\right)+b_{2} e_{2}+m_{2} m_{2}+n_{2} s_{2}+t_{1}\left(m_{2} n_{2}+n_{2} t_{2}\right) \\
& -\left(e_{2}+s_{1} n_{2}+t_{1}\left(m_{2}+t_{1} n_{2}\right)\right)+\varepsilon_{1}\left(m_{2}+t_{1} n_{2}\right)=-e_{0}=0,
\end{aligned}
$$

It follows that $e_{0}=0$. And this implies $e_{i}=0(0 \leq i \leq d-2)$. The coefficient $v_{1}$ becomes

$$
\begin{aligned}
& f_{2} n_{3}+s_{1}\left(n_{2} m_{3}+t_{2} n_{3}\right)+f_{2} n_{2}+s_{1}\left(m_{2} n_{2}+n_{2} t_{2}\right)-\left(f_{1} n_{2}+s_{1}\left(m_{2}+t_{1} n_{2}\right)\right)+\varepsilon_{1} s_{1} n_{2} \\
& =f_{0}=0 .
\end{aligned}
$$

It follows that $f_{0}=0$. And this implies $f_{i}=0(0 \leq i \leq d-2)$. So $L^{(-)} U^{(1)}=0$. Hence, $L U^{(1)} \subseteq U^{(1)}$. Since $U^{(1)}$ is invariant under $R$ and $F_{i}(0 \leq i \leq d)$, we get $U^{(1)}=V$ by Lemma 2.2, a contradiction. These complete the proof of Theorem 6.4.

7. Determine the structure of tridiagonal pairs $A, A^{*}$ of height 1 . In Section 6 , we have already displayed the shape of tridiagonal pairs of height 1 . In this section, for all cases listed in Theorem 6.4, we display a basis for $V$ and give the actions of $A, A^{*}$ on this basis.

Lemma 7.1. Suppose Theorem 6.4 (i) holds. Then

(i) $u_{0}$ is a basis for $U_{0}$,

(ii) $u_{i}, v_{i}$ is a basis for $U_{i}(1 \leq i \leq d-1)$,

(iii) $u_{d}$ is a basis for $U_{d}$,

(iv) the vectors

$$
u_{0}, u_{1}, v_{1}, \ldots, u_{d-1}, v_{d-1}, u_{d}
$$

form a basis for $V$.

Proof. Immediate from Lemmas 2.5, 4.1 and 4.4.

We now give the actions of $R, L$ on the basis in Lemma 7.1.

Lemma 7.2. Suppose Theorem 6.4(i) holds. Then there exist scalars $a_{0}, c_{1}, e_{0}$ in $\mathbb{K}$ such that the maps $R, L$ act on the basis (7.108) as follows.

$$
\begin{aligned}
& R u_{i}=u_{i+1} \quad(0 \leq i \leq d-1), \quad R u_{d}=0, \\
& R v_{i}=v_{i+1} \quad(1 \leq i \leq d-2), \quad R v_{d-1}=0, \\
& L u_{0}=0, \quad L u_{1}=a_{0} u_{0}, \quad L u_{i+1}=a_{i} u_{i}+b_{i} v_{i} \quad(1 \leq i \leq d-1), \\
& L v_{1}=e_{0} u_{0}, \quad L v_{i+1}=e_{i} u_{i}+c_{i} v_{i} \quad(1 \leq i \leq d-2),
\end{aligned}
$$

where if $d$ is odd, the coefficients satisfy (5.80), (5.81), (4.73), (4.74), (5.82), (5.83), (5.84) and (5.85); if $d$ is even, the coefficients satisfy (5.93), (5.94), (4.73), (4.74), (5.95), (5.96), (5.97) and (5.98).

Proof. Immediate from Lemmas 2.11 and 7.1, and equations (4.72), (5.75), (5.76) and (5.77). 
Theorem 7.3. Suppose Theorem 6.4(i) holds. Then A, $A^{*}$ act on the basis (7.108) as follows.

$$
\begin{aligned}
& A u_{i}=\theta_{i} u_{i}+u_{i+1} \quad(0 \leq i \leq d-1), \quad A u_{d}=\theta_{d} u_{d}, \\
& A v_{i}=\theta_{i} v_{i}+v_{i+1} \quad(1 \leq i \leq d-2), \quad A v_{d-1}=\theta_{d-1} v_{d-1}, \\
& A^{*} u_{0}=\theta_{0}^{*} u_{0}, \quad A^{*} u_{1}=a_{0} u_{0}+\theta_{1}^{*} u_{1}, \\
& A^{*} u_{i+1}=a_{i} u_{i}+b_{i} v_{i}+\theta_{i+1}^{*} u_{i+1} \quad(1 \leq i \leq d-1), \\
& A^{*} v_{1}=e_{0} u_{0}+\theta_{1}^{*} v_{1}, \quad A^{*} v_{i+1}=e_{i} u_{i}+c_{i} v_{i}+\theta_{i+1}^{*} v_{i+1} \quad(1 \leq i \leq d-2) .
\end{aligned}
$$

Proof. Immediate from Lemma 7.2 and the definition of $R, L$.

Lemma 7.4. Suppose Theorem 6.4 (ii) holds. Then

(i) $u_{0}$ is a basis for $U_{0}$,

(ii) $u_{i}, v_{i}, w_{i}$ is a basis for $U_{i}(1 \leq i \leq 2)$,

(iii) $u_{3}$ is a basis for $U_{3}$,

(iv) the vectors

$$
u_{0}, u_{1}, v_{1}, w_{1}, u_{2}, v_{2}, w_{2}, u_{3}
$$

form a basis for $V$.

Proof. Similar to the proof of Lemma 7.1.

We now give the actions of $R, L$ on the basis in Lemma 7.4.

LEMma 7.5. Suppose Theorem 6.4 (ii) holds. Then there exist scalars $a_{0}, e_{0}, f_{0}, s_{1}, t_{1}$ in $\mathbb{K}$ such that the maps $R, L$ act on the basis (7.109) as follows.

$$
\begin{aligned}
R u_{0} & =u_{1}, \quad R u_{1}=u_{2}, \quad R u_{2}=u_{3}, \quad R u_{3}=0, \\
R v_{1} & =v_{2}, \quad R v_{2}=0, \\
R w_{1} & =w_{2}, \quad R w_{2}=0, \\
L u_{0} & =0, \quad L u_{1}=a_{0} u_{0}, \quad L u_{i+1}=a_{i} u_{i}+b_{i} v_{i}(1 \leqslant i \leqslant 2), \\
L v_{1} & =e_{0} u_{0}, \quad L v_{2}=e_{1} u_{1}+w_{1}, \\
L w_{1} & =f_{0} u_{0}, \quad L w_{2}=f_{1} u_{1}+s_{1} v_{1}+t_{1} w_{1},
\end{aligned}
$$

where

$$
\begin{aligned}
& a_{1}=2(d-1) c c^{*}, \quad a_{2}=a_{0}-2\left(2 b^{*} c+2 b c^{*}+(d+3) c c^{*}\right), \\
& b_{1}=1, \quad b_{2}=-1 \\
& e_{1}=-e_{0} \\
& f_{1}=-f_{0} .
\end{aligned}
$$

Proof. Similar to the proof of Lemma 7.2. 
Theorem 7.6. Suppose Theorem 6.4 (ii) holds. Then A, $A^{*}$ act on the basis (7.109) as follows.

$$
\begin{aligned}
& A u_{i}=\theta_{i} u_{i}+u_{i+1} \quad(0 \leq i \leq 2), \quad A u_{3}=\theta_{3} u_{3}, \\
& A v_{1}=\theta_{1} v_{1}+v_{2}, \quad A v_{2}=\theta_{2} v_{2}, \\
& A^{*} u_{0}=\theta_{0}^{*} u_{0}, \quad A^{*} u_{1}=a_{0} u_{0}+\theta_{1}^{*} u_{1}, \\
& A^{*} u_{i+1}=a_{i} u_{i}+b_{i} v_{i}+\theta_{i+1}^{*} u_{i+1} \quad(1 \leq i \leq 2), \\
& A^{*} v_{1}=e_{0} u_{0}+\theta_{1}^{*} v_{1}, \quad A^{*} v_{2}=e_{1} u_{1}+w_{1}+\theta_{2}^{*} v_{2}, \\
& A^{*} \omega_{1}=f_{0} u_{0}+\theta_{1}^{*} \omega_{1}, \quad A^{*} \omega_{2}=f_{1} u_{1}+s_{1} v_{1}+t_{1} \omega_{1}+\theta_{2}^{*} \omega_{2} .
\end{aligned}
$$

Proof. Similar to the proof of Theorem 7.3.

Acknowledgment. We would like to thank an anonymous referee for his/her very careful reading and useful suggestions. This work was supported by the NSF of China (no. 11471097 and no. 11971146) and the NSF of Hebei Province (no. A2017403010 and no. A2019205089).

\section{REFERENCES}

[1] T. Ito, K. Tanabe, and P. Terwilliger. Some algebra related to $P$-and $Q$-polynomial association schemes. Codes and Association Schemes (Piscataway, NJ, 1999), American Mathematical Society, Providence, 167-192, 2001.

[2] T. Ito, K. Nomura, and P. Terwilliger. A classification of sharp tridiagonal pairs. Linear Algebra and its Applications, 435:1857-1884, 2011.

[3] K. Nomura. A refinement of the split decomposition of a tridiagonal pair. Linear Algebra and its Applications, 403:1-23, 2005.

[4] K. Nomura. Tridiagonal pairs of height one. Linear Algebra and its Applications, 403:118-142, 2005.

[5] K. Nomura and P. Terwilliger. Affine transformations of a Leonard pair. Electronic Journal of Linear Algebra, 16: 389-418, 2007.

[6] K. Nomura and P. Terwilliger. Sharp tridiagonal pairs. Linear Algebra and its Applications, 429:79-99, 2008.

[7] K. Nomura and P. Terwilliger. The structure of a tridiagonal pair. Linear Algebra and its Applications, 429:1647-1662, 2008.

[8] P. Terwilliger. Two linear transformations each tridiagonal with respect to an eigenbasis of the other. Linear Algebra and its Applications, 330:149-203, 2001.

[9] P. Terwilliger. Leonard pairs from 24 points of view. Rocky Mountain Journal of Mathematics, 32:827-888, 2002. 